\title{
Joint Transmit Precoding and Reconfigurable Intelligent Surface Phase Adjustment: A Decomposition-Aided Channel Estimation Approach
}

\author{
Zhengyi Zhou, Ning Ge, Member, IEEE, \\ Zhaocheng Wang, Senior Member, IEEE, and Lajos Hanzo, Fellow, IEEE
}

\begin{abstract}
Reconfigurable intelligent surfaces (RISs), consisting of many low-cost elements that reflect the incident waves by an adjustable phase shift, have attracted sudden attention for their potential of reconfiguring the signal propagation environment and enhancing the performance of wireless networks. The passive nature of RISs is indeed beneficial, but the lack of radio frequency (RF) chains at the RIS has made channel estimation extremely challenging. We face this challenge by proposing a joint channel estimation and transmit precoding framework for RIS-aided multiple-input multiple-output (MIMO) systems. Specifically, the effective cascaded channel of the reflected transmitter-RISreceiver link is decomposed into multiple subchannels, each of which corresponds to a single RIS element. Then our joint RIStransmitter precoding model is formulated for the individual subchannels of each reflecting element. Finally, we develop a twostage precoding design for successively determining the required phase shifts of each reflecting element of the RIS and the digital baseband precoder of the transmitter, only relying on the channel state information (CSI) of the subchannels. The performance of the proposed subchannel estimation and joint precoding method is evaluated by extensive simulations. Our numerical results show that the proposed designs provide an attractive solution to RISaided MIMO systems.
\end{abstract}

Index Terms-Reconfigurable intelligent surface, channel estimation, precoding, MIMO, channel decomposition.

\section{INTRODUCTION}

$\mathbf{T}$ HE last decade has witnessed the tremendous development of wireless communications. The wireless teletraffic has been escalating, and is projected to be as high as 77 exabytes per month by 2022 [1], [2]. Although the spectral efficiency of wireless systems has been substantially improved by various technological advances, including massive multiple-input multiple-output (MIMO), millimeter wave (mmWave) communications, etc, it is desirable to reduce both

This work was supported in part by the National Key R\&D Program of China under Grant 2018YFA0701601, and in part by the National Natural Science Foundation of China (Grant No. 91638205). L. Hanzo would like to acknowledge the financial support of the Engineering and Physical Sciences Research Council projects EP/N004558/1, EP/P034284/1, EP/P034284/1, EP/P003990/1 (COALESCE), of the Royal Society's Global Challenges Research Fund Grant as well as of the European Research Council's Advanced Fellow Grant QuantCom. (Corresponding author: Zhaocheng Wang.)

Z. Zhou, Ning Ge, and Z. Wang are with Beijing National Research Center for Information Science and Technology (BNRist), Department of Electronic Engineering, Tsinghua University, Beijing 100084, China (E-mails: zhou-zy16@mails.tsinghua.edu.cn; gening@tsinghua.edu.cn; zcwang@mail.tsinghua.edu.cn).

Z. Zhou N. Ge, and Z. Wang are with Beijing National Research Center for Information Science and Technology (BNRist), Departmen$\mathrm{t}$ of Electronic Engineering, Tsinghua University, Beijing 100084, China. E-mails: zhou-zy16@mails.tsinghua.edu.cn; gening@tsinghua.edu.cn; zcwang@tsinghua.edu.cn.

L. Hanzo is with the School of Electronics and Computer Science, University of Southampton, Southampton, SO17 1BJ, UK. E-mail: 1h@ecs.soton.ac.uk. the required high hardware implementation cost and the energy consumption [3].

Recently, the new concept of reconfigurable intelligent surfaces (RISs) has emerged and has been adopted for wireless communication [4], [5]. RISs, also referred to as intelligent reflecting surfaces (IRSs), rely on man-made planar arrays that consist of numerous low-cost passive elements. More explicitly, based on reconfigurable electromagnetic (EM) material, each of the elements is able to reflect the incident waves by an adjustable phase shift. Conventionally, RISs have been used to implement reconfigurable reflect-array antennas by illuminating the nearby reflecting surface with the aid of active antennas at the transmitter [6]-[8]. However, it has also been proposed to place the RIS somewhere between the transmitter and the receiver, so that a reflected transmitter-RIS-receiver link is established in addition to the direct transmitter-receiver link. In this manner, the signal propagation can be conveniently reconfigured by appropriately modifying the phase shift of the reflecting elements (REs), without requiring any extra power for complex signal processing or retransmission operations. As a benefit, the performance of wireless systems is expected to be considerably enhanced by adaptively "adjusting" the wireless propagation environment [9]-[11].

In order to realize the attractive potential of RISs, the state-of-the-art studies aimed to jointly optimize the baseband digital precoder of the transmitter and the phase shifts of the RIS elements. Most contributions focused on the RISaided multi-user multiple-input-single-output scenario [12][15]. Specifically, the transmission power consumption was minimized under the users' individual communication rate constraints [12]. In [13], the weighted sum-rate of the users was maximized with the aid of fractional programming. The authors of [14] considered the practical hardware limitations and optimized the achievable rate using limited discrete phase shifts of the REs. The benefits of the RIS are investigated in multigroup multicast systems by alternately solving a pair of second-order cone programming (SOCP) problems [15]. As for RIS-aided MIMO scenarios, the performance metrics of both channel capacity [16], [17] and symbol error rate [18] are studied for point-to-point MIMO systems, while the associated sum-rate is maximized in multi-cell MIMO systems [19]. It is important to note that all the above-mentioned investigations have assumed perfect knowledge of the channels involved in both the direct and the reflected links. However, channel estimation is a challenging issue for RIS-aided systems. As discussed in [20], most of current research works assume that the nearly-passive RISs are not equipped with sensing 
elements or radio frequency $(\mathrm{RF})$ chains,${ }^{1}$ and therefore, it is impractical to directly estimate the channels of the reflected link, i.e., the incident channel spanning from the transmitter to the RIS, and the reflected channel spanning from the RIS to the receiver. ${ }^{2}$ Without accurate channel state information (CSI) of the reflected link, using the existing precoding designs may not be feasible, which thus limits the practical applications of RISs.

At present, most channel estimation related works considered single-antenna receivers in RIS-aided systems [24]-[28]. The basic methodology adopted by these studies is to estimate the equivalent cascaded channel involved in the reflected link, instead of estimating the incident and the reflected channels. However, since the cascaded channels are routinely defined with respect to single receiver antennas, the majority of current research has been focused on multiple-input-singleoutput (MISO) scenarios, while precoding designs have not yet been considered relying on the cascaded CSI for pointto-point RIS-aided MIMO systems. As for the scenario of MIMO communications, the channels involved in the reflected link are typically estimated based on the methodology of sparse matrix factorization and completion, using techniques such as message passing [29], [30], and compressive sensing [31], which are however only appropriate for sparse channel conditions and impose a potentially high signal processing complexity. Therefore, there is a paucity of solutions for RISaided MIMO systems, which thus motivates this contribution.

In this paper, a feasible channel estimation and joint precoding framework is proposed for RIS-aided MIMO communication systems, where both the transmitter and the receiver are equipped with multiple antennas. In lieu of estimating the incident and the reflected channels directly, we decompose the effective channel of the reflected link into multiple subchannels, and solve the channel estimation as well as joint precoding problems for each of the subchannels. In contrast to the cascaded channels that are defined with respect to the receiver antennas, such as in [27], [32], every subchannel corresponds to a specific RIS reflecting element which constitutes a separate transmitter-RIS-receiver reflecting path. Given its distinct physical interpretation as well as mathematical formulation, a series of new precoding methods can be derived based on this subchannel model. Our main contributions are summarized as follows.

- A channel decomposition model is proposed for RISaided MIMO communications. The effective channel of the reflected link is decomposed into multiple subchannels, each of which corresponds to a single RIS element. We show that it is feasible to estimate the subchannels,

\footnotetext{
${ }^{1}$ Surfaces with active elements, namely that RF circuits and signal processing units are embedded in the surface, are generally referred to as active Holographic Multiple Input Multiple Output Surface (HMIMOS), or Large Intelligent Surface (LIS) [21]. For an active HMIMOS, conventional channel estimation methods developed for MIMO systems can be readily applied.

${ }^{2}$ Recently, there are also some works on RIS-based architectures that include active elements for channel estimation. In [22], the authors investigate an architecture, where the RIS is equipped with a few elements that are active and connected to the baseband of the RIS controller. The authors of [23] consider a RIS comprising a single RF chain used for baseband measurements, in addition to a controller and many passive elements.
}

and exploit their knowledge for joint precoding without performance loss.

- We develop a subchannel estimation mechanism based on multi-round pilot training, where the REs of the RIS configure the phase shifts in each round according to a preset codebook. A sufficient condition is derived for the codebook to minimize the mean square error (MSE) of the subchannel estimator, and then both the Hadamard matrix based and the discrete Fourier transform (DFT) aided codebooks are used for practical implementations.

- We reformulate the joint RIS-transmitter precoding problem based on the above-mentioned channel decomposition model, wherein the digital precoder of the transmitter and the phase shifts of the REs can be designed by purely relying on the CSI of the subchannels. In this way, joint precoding becomes feasible in practical systems.

- A two-stage precoding design is proposed based on only the knowledge of the subchannels. Firstly, the reflecting phase shifts are determined by solving an approximation of the original problem using a fixed-point based method. After that, the baseband precoder of the transmitter is optimized given the phase shifts.

- We evaluate the performance of the proposed channel estimation and joint precoding methods for different simulation settings, including the transmission power constraint, the number of REs, and channel propagation conditions. Our numerical results show that the channel decomposition based framework provides a viable solution for RIS-aided MIMO systems.

Noting that an alternating optimization (AO) method is also developed for RIS-aided point-to-point MIMO systems [17], we employ this design as a benchmark, and show that our proposed precoding scheme has advantages over its AO-based counterpart.

The rest of this paper is organized as follows. In Section II, we introduce the system model, while in Section III, we define the subchannels and propose the subchannel estimation method. In Section IV, our precoding technique is designed purely relying on the CSI of the subchannels. Numerical results are presented in Section $\mathrm{V}$ and the paper is concluded in Section VI.

The following notations are adopted throughout this paper. $\mathbb{C}^{n}$ denotes the $n$-dimensional complex space, and $\mathbb{C}^{m \times n}$ is the space of $m \times n$ complex matrices. $\boldsymbol{A}$ is a matrix and $\boldsymbol{a}$ is a vector. The transpose, conjugate transpose and inverse operators are denoted by $(\cdot)^{\mathrm{T}},(\cdot)^{\mathrm{H}}$ and $(\cdot)^{-1}$, respectively. $\operatorname{tr}(\cdot)$ is the trace operator, and $\operatorname{rank}(\cdot)$ is the rank of a matrix. The operator $|\cdot|$ denotes the magnitude for complex numbers, the entry-wise magnitude for vectors, and the determinant for matrices. $\|\cdot\|_{1},\|\cdot\|_{2}$ and $\|\cdot\|_{F}$ denote the 1-norm, 2norm and Frobenius norm, respectively. $[\boldsymbol{A}]_{\left(m_{1}: m_{2}, n_{1}: n_{2}\right)}$ is the corresponding subcomponent of $\boldsymbol{A}$, with row indices spanning from $m_{1}$ to $m_{2}$, and column indices spanning from $n_{1}$ to $n_{2}$. The diagonal matrix with diagonal entries $\left\{a_{1}, \cdots, a_{n}\right\}$ is denoted by $\operatorname{diag}\left\{a_{1}, \cdots, a_{n}\right\}, \boldsymbol{I}_{n}$ is the $n \times n$ identity matrix, $\mathbf{0}_{n}$ is the $n$-dimensional all-zero vector, and $\mathbf{0}$ denotes zero vector or matrix of appropriate dimension. A complex number $a \in \mathbb{C}$ is represented by $a=|a| e^{\mathrm{j} \angle a}$. We use $\otimes$ for 


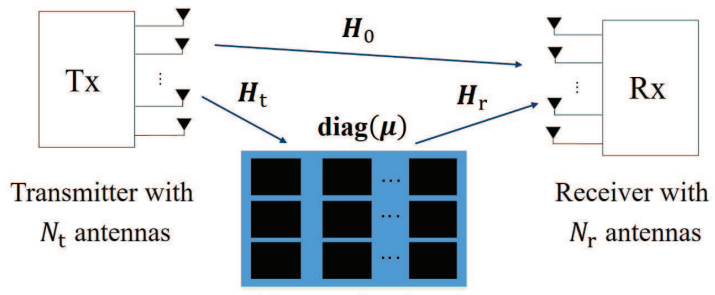

RIS with $M$ reflecting elements

Fig. 1. A RIS-aided point-to-point MIMO system, where the transmitter and the receiver are equipped with $N_{\mathrm{t}}$ and $N_{\mathrm{r}}$ antennas, respectively, and the RIS employs $M$ REs.

the Kronecker product, $\operatorname{vec}(\cdot)$ for the vectorization operation, and $E\{\cdot\}$ for the expectation operator, respectively.

\section{SYSTEM MODEL}

\section{A. Signal Model}

We consider the RIS-aided point-to-point MIMO system, as illustrated in Fig. 1, where the transmitter employs $N_{\mathrm{t}}$ transmit antennas (TAs) to forward $N_{\mathrm{s}}$ data streams to the receiver equipped with $N_{\mathrm{r}}$ receiving antennas (RAs), with $N_{\mathrm{s}} \leq \min \left\{N_{\mathrm{t}}, N_{\mathrm{r}}\right\}$. The RIS consists of $M$ REs that can modify the incident signal by an adjustable phase shift, and is coordinated by the transmitter using a wireless or wired control link. The signals from both the direct transmitter-receiver link and the reflected transmitter-RIS-receiver link are superposed at the receiver.

The transmitter applies an $N_{\mathrm{t}} \times N_{\mathrm{s}}$ baseband precoder $\boldsymbol{W}$ for transmitting an $N_{\mathrm{s}} \times 1$ symbol vector $s$, with $\mathrm{E}\left\{s s^{\mathrm{H}}\right\}=$ $\rho \boldsymbol{I}_{N_{\mathrm{s}}}$, where $\rho$ is the total transmission power, and the precoder is normalized such that $\|\boldsymbol{W}\|_{F}^{2} \leq 1$ to enforce the power constraint. The $m$ th element of the RIS reflects the signal from the transmitter with a phase shift of $\omega_{m} \in[0,2 \pi)(1 \leq m \leq$ $M)$. Given the narrowband block-fading channel model, the $N_{\mathrm{r}} \times 1$ received signal is obtained as

$$
\begin{aligned}
\boldsymbol{y} & =\underbrace{\boldsymbol{H}_{0} \boldsymbol{W} \boldsymbol{s}}_{\text {Direct link }}+\underbrace{\boldsymbol{H}_{\mathrm{r}} \operatorname{diag}(\boldsymbol{\mu}) \boldsymbol{H}_{\mathrm{t}} \boldsymbol{W} \boldsymbol{s}}_{\text {Reflected link }}+\boldsymbol{z} \\
& =\left(\boldsymbol{H}_{0}+\boldsymbol{H}_{\mathrm{r}} \operatorname{diag}(\boldsymbol{\mu}) \boldsymbol{H}_{\mathrm{t}}\right) \boldsymbol{W} \boldsymbol{s}+\boldsymbol{z},
\end{aligned}
$$

where $\boldsymbol{\mu}=e^{\mathrm{j} \boldsymbol{\omega}}=\left[e^{\mathrm{j} \omega_{1}}, \ldots, e^{\mathrm{j} \omega_{M}}\right]^{\mathrm{T}}$ is the $M \times 1$ phase shift vector, $\boldsymbol{z} \in \mathbb{C}^{N_{\mathrm{r}}}$ is the noise vector following $\boldsymbol{z} \sim$ $\mathcal{C N}\left(\mathbf{0}_{N_{\mathrm{r}}}, \sigma^{2} \boldsymbol{I}_{N_{\mathrm{r}}}\right)$. Furthermore, $\boldsymbol{H}_{0} \in \mathbb{C}^{N_{\mathrm{r}} \times N_{\mathrm{t}}}$ is the direct channel from the transmitter to the receiver, $\boldsymbol{H}_{\mathrm{t}} \in \mathbb{C}^{M \times N_{\mathrm{t}}}$ is the incident channel from the transmitter to the RIS, and finally $\boldsymbol{H}_{\mathrm{r}} \in \mathbb{C}^{N_{\mathrm{r}} \times M}$ is the reflected channel from the RIS to the receiver. All the channels involved are assumed to be quasistatic, i.e., approximately constant during both estimation and precoding. With the subscripts omitted for simplicity, the channels are expressed as $\boldsymbol{H}=\beta \overline{\boldsymbol{H}}$, where $\beta$ is the path loss, and $\overline{\boldsymbol{H}}$ is the small-scale Rician fading given by [12], [17], [34] $]^{3}$

$$
\overline{\boldsymbol{H}}=\sqrt{\frac{K}{1+K}} e^{\mathrm{j} \Omega} \overline{\boldsymbol{H}}^{(\mathrm{LOS})}+\sqrt{\frac{1}{1+K}} \overline{\boldsymbol{H}}^{(\mathrm{NLOS})},
$$

\footnotetext{
${ }^{3}$ The channel model of (2) is used for numerical simulation. However, the proposed subchannel estimation and precoding methods are not limited to this model and can be applied to general channel models without any extra requirements.
}

where $K$ is the Rician factor, $\overline{\boldsymbol{H}}^{(\mathrm{LOS})}$ is the line-of-sight (LOS) component, $\overline{\boldsymbol{H}}^{\text {(NLOS) }}$ is the non-LOS (NLOS) component, and $\Omega$ is a random phase shift. ${ }^{4}$ The columns of $\overline{\boldsymbol{H}}^{\text {(NLOS) }}$ follow the complex symmetric Gaussian distribution with a zero mean and a covariance matrix of $\boldsymbol{R}$, i.e. $\mathcal{C N}(\mathbf{0}, \boldsymbol{R}){ }^{5}$ while $\overline{\boldsymbol{H}}^{(\mathrm{LOS})}$ is expressed as $\overline{\boldsymbol{H}}^{(\mathrm{LOS})}=$ $\boldsymbol{a}_{\mathrm{r}}\left(\phi^{(\mathrm{r})}, \varphi^{(\mathrm{r})}\right) \boldsymbol{a}_{\mathrm{t}}^{\mathrm{H}}\left(\phi^{(\mathrm{t})}, \varphi^{(\mathrm{t})}\right)$, where $\phi^{(\mathrm{r})}\left(\varphi^{(\mathrm{r})}\right)$ and $\phi^{(\mathrm{t})}\left(\varphi^{(\mathrm{t})}\right)$ are the azimuth (elevation) angles of arrival and departure (AOA and AOD), and $\boldsymbol{a}_{\mathrm{r}}(\phi, \varphi)\left(\boldsymbol{a}_{\mathrm{t}}(\phi, \varphi)\right)$ are the TA (RA) array response vectors. For an $N_{\mathrm{H}} \times N_{\mathrm{V}}$ uniform planar array (UPA), the array response vector $\boldsymbol{a}_{\mathrm{UPA}}(\phi, \varphi)$ is expressed as

$$
\begin{aligned}
\boldsymbol{a}_{\mathrm{UPA}}(\phi, \varphi) & =\frac{1}{\sqrt{N_{\mathrm{H}} N_{\mathrm{V}}}}\left[1 \cdots e^{\mathrm{j} \frac{2 \pi}{\lambda} D(h \sin \phi \sin \varphi+v \cos \varphi)}\right. \\
& \left.\cdots e^{\mathrm{j} \frac{2 \pi}{\lambda} D\left(\left(N_{\mathrm{H}}-1\right) \sin \phi \sin \varphi+\left(N_{\mathrm{V}}-1\right) \cos \varphi\right)}\right]^{\mathrm{T}},
\end{aligned}
$$

where $\lambda$ is the transmission wavelength, and $D$ is the spacing between adjacent array elements, $0 \leq h \leq\left(N_{\mathrm{H}}-1\right)$, and $0 \leq v \leq\left(N_{\mathrm{V}}-1\right)$.

\section{B. Pilot Training Model}

The transmitter sends the pilot signal $\boldsymbol{X}=$ $\left[\boldsymbol{x}_{1}^{\mathrm{T}}, \boldsymbol{x}_{2}^{\mathrm{T}}, \ldots, \boldsymbol{x}_{N_{\mathrm{t}}}^{\mathrm{T}}\right]^{\mathrm{T}} \in \mathbb{C}^{N_{\mathrm{t}} \times \tau}$ to the receiver, where $\boldsymbol{x}_{n} \in \mathbb{C}^{1 \times \tau}, 1 \leq n \leq N_{\mathrm{t}}$ is the pilot of length $\tau$, loaded on the $n$th antenna element. The pilot matrix is normalized such that $\|\boldsymbol{X}\|_{F}^{2}=\tau \rho$. Given the phase shifts adjusted by the REs of the RIS during the transmission of $\boldsymbol{X}$, the receiver sees an $N_{\mathrm{r}} \times N_{\mathrm{t}}$ effective channel of

$$
\boldsymbol{H}_{\mathrm{e}}(\boldsymbol{\mu})=\boldsymbol{H}_{0}+\boldsymbol{H}_{\mathrm{r}} \operatorname{diag}(\boldsymbol{\mu}) \boldsymbol{H}_{\mathrm{t}} .
$$

The $N_{\mathrm{r}} \times \tau$ received signal is then expressed as $\boldsymbol{Y}=$ $\boldsymbol{H}_{\mathrm{e}}(\boldsymbol{\mu}) \boldsymbol{X}+\boldsymbol{Z}$, where $\boldsymbol{Z} \in \mathbb{C}^{N_{\mathrm{r}} \times \tau}$ is the noise matrix whose column vectors follow the same distribution as $z$ in (1). ${ }^{6}$ In general, orthogonal pilots are employed, and the length of the pilot signal is designed such that $\tau \geq N_{\mathrm{t}}$. Thus, the effective channel can be estimated using the typical least-square (LS) algorithm:

$$
\hat{\boldsymbol{H}}_{\mathrm{e}}(\boldsymbol{\mu})=\boldsymbol{Y} \boldsymbol{X}^{\mathrm{H}}\left(\boldsymbol{X} \boldsymbol{X}^{\mathrm{H}}\right)^{-1}=\boldsymbol{H}_{\mathrm{e}}(\boldsymbol{\mu})+\widetilde{\boldsymbol{Z}},
$$

where $\widetilde{\boldsymbol{Z}}=\boldsymbol{Z} \boldsymbol{X}^{\mathrm{H}}\left(\boldsymbol{X} \boldsymbol{X}^{\mathrm{H}}\right)^{-1} \in \mathbb{C}^{N_{\mathrm{r}} \times N_{\mathrm{t}}}$ is the effective noise, and $\hat{\boldsymbol{H}}_{\mathrm{e}}(\boldsymbol{\mu}) \in \mathbb{C}^{N_{\mathrm{r}} \times N_{\mathrm{t}}}$ is the estimate of $\boldsymbol{H}_{\mathrm{e}}(\boldsymbol{\mu})$. As shown in (4)-(5), the full CSI of $\boldsymbol{H}_{\mathrm{r}}$ and $\boldsymbol{H}_{\mathrm{t}}$, required by most current precoding methods, cannot be readily acquired using

\footnotetext{
${ }^{4}$ As discussed in [34], the phase shift in the LoS part is uniformly distributed on $[0,2 \pi)$, and varies in each channel realization. However, the derivation of our proposed estimation and precoding schemes does not rely on any specific channel model.

${ }^{5}$ According to [35], the Rayleigh fading channel is actually spatially correlated for receivers equipped with planar arrays. Given $N_{\mathrm{H}} \times N_{\mathrm{V}}$ uniform planar arrays with inter-element spacing of $D$, the entries of the covariance matrix is determined by $[\boldsymbol{R}]_{n, m}=\gamma \frac{\sin \left(2 \pi\left\|\boldsymbol{u}_{n}-\boldsymbol{u}_{m}\right\|_{2} D / \lambda\right)}{2 \pi\left\|\boldsymbol{u}_{n}-\boldsymbol{u}_{m}\right\|_{2} D / \lambda}$, where $\lambda$ is the carrier wavelength, $\gamma$ is a normalizing factor, and $\boldsymbol{u}_{n}=[0, i(n), j(n)]^{\mathrm{T}}$ with $i(n)=\bmod \left(n-1, N_{\mathrm{H}}\right)$ and $j(n)=\left\lfloor(n-1) / N_{\mathrm{V}}\right\rfloor$.

${ }^{6}$ For single-antenna receivers, the reflected channel becomes $\boldsymbol{h}_{\mathrm{r}} \in \mathbb{C}^{M}$. The equivalent cascaded channel is then defined as $\boldsymbol{H}_{\text {cas }}=\boldsymbol{d i a g}\left(\boldsymbol{h}_{\mathrm{r}}^{\mathrm{H}}\right) \boldsymbol{H}_{\mathrm{t}}$ such that $\boldsymbol{h}_{\mathrm{r}}^{\mathrm{H}} \operatorname{diag}(\boldsymbol{\mu}) \boldsymbol{H}_{\mathrm{t}}=\boldsymbol{\mu}^{\mathrm{T}} \boldsymbol{H}_{\text {cas }}$. Most existing studies have been focused on the estimation of $\boldsymbol{H}_{\text {cas }}$, and have considered MISO scenarios based on the cascaded channels, while precoding designs using the cascaded CSI for multi-antenna receivers have not yet been investigated in the open literature.
} 
conventional pilot-aided training. The only available knowledge of $\hat{\boldsymbol{H}}_{\mathrm{e}}(\boldsymbol{\mu})$ acquired from pilot-aided training will limit the practical applications of the existing precoding designs. In the next section, instead of estimating $\boldsymbol{H}_{\mathrm{r}}$ and $\boldsymbol{H}_{\mathrm{t}}$, we propose to decompose the effective channel into multiple subchannels and estimate the subchannels by repeating the pilot training a number of times and beneficially adjusting the RIS phase shifts.

\section{Channel Decomposition and Subchannel ESTIMATION}

In this section, we detail the proposed channeldecomposition-based subchannel estimation methods. Firstly, the effective channel of (4) is decomposed into multiple subchannels, each of which corresponds to a single RIS RE. Afterwards, we show that the subchannels can be estimated using multi-round pilot training, by appropriately configuring the reflecting phase shifts in each training round. Moreover, the optimal phase adjustment is designed by minimizing the MSE of the subchannel estimator based on both the Hadamard matrix and the DFT matrix. Finally, we provide a performance analysis of the proposed method.

\section{A. Channel Decomposition Based Subchannel Estimation}

Note that the incident and the reflected channels, $\boldsymbol{H}_{\mathrm{r}}$ and $\boldsymbol{H}_{\mathrm{t}}$, cannot be directly estimated due to the lack of RF chains at the RIS. However, the information concerning $\boldsymbol{H}_{\mathrm{r}}$ and $\boldsymbol{H}_{\mathrm{t}}$ can still be inferred from the effective channel $\boldsymbol{H}_{\mathrm{e}}$. Specifically, $\boldsymbol{H}_{\mathrm{e}}$ can be decomposed as follows

$$
\begin{aligned}
\boldsymbol{H}_{\mathrm{e}}(\boldsymbol{\mu}) & =\boldsymbol{H}_{0}+\sum_{m=1}^{M} \mu_{m}\left[\boldsymbol{H}_{\mathrm{r}}\right]_{(:, m)}\left[\boldsymbol{H}_{\mathrm{t}}\right]_{(m,:)} \\
& =\boldsymbol{H}_{0}+\sum_{m=1}^{M} \mu_{m} \boldsymbol{H}_{m},
\end{aligned}
$$

where $\mu_{m}=e^{\mathrm{j} \omega_{m}}$ is the $m$ th entry of the phase shift vector, $\left[\boldsymbol{H}_{\mathrm{r}}\right]_{(:, m)}$ is the $m$ th column of the reflected channel $\boldsymbol{H}_{\mathrm{r}}$, $\left[\boldsymbol{H}_{\mathrm{t}}\right]_{(m,:)}$ is the $m$ th row of the incident channel $\boldsymbol{H}_{\mathrm{t}}$, and $\boldsymbol{H}_{m} \triangleq\left[\boldsymbol{H}_{\mathrm{r}}\right]_{(:, m)}\left[\boldsymbol{H}_{\mathrm{t}}\right]_{(m,:)} \in \mathbb{C}^{N_{\mathrm{r}} \times N_{\mathrm{t}}}$ is defined as the $m$ th subchannel of $\boldsymbol{H}_{\mathrm{e}}$, for $1 \leq m \leq M$. By defining the augmented phase shift vector as $\overline{\boldsymbol{\mu}}=\left[1, \boldsymbol{\mu}^{\mathrm{T}}\right]^{\mathrm{T}}$, we can rewrite $\boldsymbol{H}_{\mathrm{e}}(\boldsymbol{\mu})$ as

$$
\boldsymbol{H}_{\mathrm{e}}(\boldsymbol{\mu})=\left(\overline{\boldsymbol{\mu}}^{\mathrm{T}} \otimes \boldsymbol{I}_{N_{\mathrm{r}}}\right)\left[\boldsymbol{H}_{0}^{\mathrm{T}}, \boldsymbol{H}_{1}^{\mathrm{T}}, \ldots, \boldsymbol{H}_{M}^{\mathrm{T}}\right]^{\mathrm{T}} .
$$

Noting that different effective channels $\boldsymbol{H}_{\mathrm{e}}(\boldsymbol{\mu})$ can be derived by varying the phase shift vector $\boldsymbol{\mu}$, we now show that the $M+1$ subchannels, $\boldsymbol{H}_{m}$, for $0 \leq m \leq M$, can be estimated via multiple observations of $\boldsymbol{H}_{\mathrm{e}}(\boldsymbol{\mu})$ obtained by repeating the LS algorithm of (5) for $L(L \geq M+1)$ times.

Based on (5) and (7), let $\overline{\boldsymbol{\mu}}_{l}=\left[1, \boldsymbol{\mu}_{l}^{\mathrm{T}}\right]^{\mathrm{T}}, 1 \leq l \leq L$ be the augmented phase shift vector configured by the RIS in the $l$ th round of training. Then the LS algorithm yields

$$
\begin{aligned}
\hat{\boldsymbol{H}}_{\mathrm{e}, l} & =\boldsymbol{H}_{\mathrm{e}}\left(\boldsymbol{\mu}_{l}\right)+\widetilde{\boldsymbol{Z}}_{l} \\
& =\left(\overline{\boldsymbol{\mu}}_{l}^{\mathrm{T}} \otimes \boldsymbol{I}_{N_{\mathrm{r}}}\right) \cdot\left[\boldsymbol{H}_{0}^{\mathrm{T}}, \boldsymbol{H}_{1}^{\mathrm{T}}, \ldots, \boldsymbol{H}_{M}^{\mathrm{T}}\right]^{\mathrm{T}}+\widetilde{\boldsymbol{Z}}_{l},
\end{aligned}
$$

where $\hat{\boldsymbol{H}}_{\mathrm{e}, l} \in \mathbb{C}^{N_{\mathrm{r}} \times N_{\mathrm{t}}}$ is the observed effective channel, and $\widetilde{Z}_{l} \in \mathbb{C}^{N_{\mathrm{r}} \times N_{\mathrm{t}}}$ is the effective noise in the $l$ th training round. By implementing the overall $L$ rounds of LS training, and defining $\boldsymbol{\Sigma} \triangleq\left[\overline{\boldsymbol{\mu}}_{1}^{*}, \ldots, \overline{\boldsymbol{\mu}}_{L}^{*}\right] \in \mathbb{C}^{(M+1) \times L}$, we have the following observations of $\hat{\boldsymbol{H}}_{\mathrm{e}, l}$ that can be used for estimating the subchannels $\boldsymbol{H}_{m}$ :

$$
\left[\begin{array}{c}
\hat{\boldsymbol{H}}_{\mathrm{e}, 1} \\
\vdots \\
\hat{\boldsymbol{H}}_{\mathrm{e}, L}
\end{array}\right]=\left(\boldsymbol{\Sigma}^{\mathrm{H}} \otimes \boldsymbol{I}_{N_{\mathrm{r}}}\right) \cdot\left[\begin{array}{c}
\boldsymbol{H}_{0} \\
\vdots \\
\boldsymbol{H}_{M}
\end{array}\right]+\left[\begin{array}{c}
\widetilde{\boldsymbol{Z}}_{1} \\
\vdots \\
\tilde{\boldsymbol{Z}}_{L}
\end{array}\right],
$$

It is seen from (9) that, by beneficially choosing $\overline{\boldsymbol{\mu}}_{l}$ to ensure that $\boldsymbol{\Sigma}$ has full row rank, i.e., $\operatorname{rank}\left(\boldsymbol{\Sigma} \boldsymbol{\Sigma}^{\mathrm{H}}\right)=M+1$, the subchannels can be estimated by the classic LS algorithm, yielding:

$$
\begin{aligned}
{\left[\begin{array}{c}
\hat{\boldsymbol{H}}_{0} \\
\vdots \\
\hat{\boldsymbol{H}}_{M}
\end{array}\right] } & =\left(\left(\left(\boldsymbol{\Sigma} \boldsymbol{\Sigma}^{\mathrm{H}}\right)^{-1} \boldsymbol{\Sigma}\right) \otimes \boldsymbol{I}_{N_{\mathrm{r}}}\right) \cdot\left[\begin{array}{c}
\hat{\boldsymbol{H}}_{\mathrm{e}, 1} \\
\vdots \\
\hat{\boldsymbol{H}}_{\mathrm{e}, L}
\end{array}\right] \\
= & {\left[\begin{array}{c}
\boldsymbol{H}_{0} \\
\vdots \\
\boldsymbol{H}_{M}
\end{array}\right]+\left[\begin{array}{c}
\widehat{\boldsymbol{Z}}_{0} \\
\vdots \\
\hat{\boldsymbol{Z}}_{M}
\end{array}\right], }
\end{aligned}
$$

where $\hat{\boldsymbol{H}}_{m}$ is the estimate of $\boldsymbol{H}_{m}, 0 \leq m \leq M$, and

$$
\left[\begin{array}{c}
\widehat{\boldsymbol{Z}}_{0} \\
\vdots \\
\widehat{\boldsymbol{Z}}_{M}
\end{array}\right]=\left(\left(\left(\boldsymbol{\Sigma} \boldsymbol{\Sigma}^{\mathrm{H}}\right)^{-1} \boldsymbol{\Sigma}\right) \otimes \boldsymbol{I}_{N_{\mathrm{r}}}\right) \cdot\left[\begin{array}{c}
\widetilde{\boldsymbol{Z}}_{1}^{\mathrm{T}} \\
\vdots \\
\widetilde{\boldsymbol{Z}}_{L}^{\mathrm{T}}
\end{array}\right]
$$

is the equivalent noise. ${ }^{7}$ By denoting $\hat{\boldsymbol{H}}_{s}=$ $\left[\begin{array}{lll}\hat{\boldsymbol{H}}_{0}^{\mathrm{T}}, & \ldots, & \hat{\boldsymbol{H}}_{M}^{\mathrm{T}}\end{array}\right]^{\mathrm{T}}, \quad \boldsymbol{H}_{s} \quad=\left[\begin{array}{llll}\boldsymbol{H}_{0}^{\mathrm{T}}, & \ldots, & \boldsymbol{H}_{M}^{\mathrm{T}}\end{array}\right]^{\mathrm{T}}$, $\widehat{\boldsymbol{\Xi}}=\left[\begin{array}{lll}\widehat{\boldsymbol{Z}}_{0}^{\mathrm{T}}, & \ldots, & \widehat{\boldsymbol{Z}}_{M}^{\mathrm{T}}\end{array}\right]^{\mathrm{T}}$, and $\widetilde{\boldsymbol{\Xi}}=\left[\begin{array}{lll}\widetilde{\boldsymbol{Z}}_{1}^{\mathrm{T}}, \ldots, & \ldots & \boldsymbol{Z}_{L}^{\mathrm{T}}\end{array}\right]^{\mathrm{T}}$ $\left(\hat{\boldsymbol{H}}_{s}, \boldsymbol{H}_{s}, \widehat{\boldsymbol{\Xi}} \in \mathbb{C}^{(M+1) N_{\mathrm{r}} \times N_{\mathrm{t}}}\right.$, and $\left.\widetilde{\boldsymbol{\Xi}} \in \mathbb{C}^{L N_{\mathrm{r}} \times N_{\mathrm{t}}}\right)$, the MSE of $\hat{\boldsymbol{H}}_{s}$ is obtained as

$$
\begin{aligned}
\operatorname{MSE}\left(\hat{\boldsymbol{H}}_{s}\right) & =\mathrm{E}\left(\left\|\hat{\boldsymbol{H}}_{s}-\boldsymbol{H}_{s}\right\|_{F}^{2}\right)=\mathrm{E}\left(\|\widehat{\boldsymbol{\Xi}}\|_{F}^{2}\right) \\
& =\mathrm{E}\left(\left\|\left[\left(\left(\boldsymbol{\Sigma} \boldsymbol{\Sigma}^{\mathrm{H}}\right)^{-1} \boldsymbol{\Sigma}\right) \otimes \boldsymbol{I}_{N_{\mathrm{r}}}\right] \widetilde{\boldsymbol{\Xi}}\right\|_{F}^{2}\right) .
\end{aligned}
$$

Thus, the MSE of the subchannel estimator can be minimized by appropriately designing $\boldsymbol{\Sigma}$.

The pilot pattern of the proposed subchannel estimation method is presented in Fig. 2, where the $N_{\mathrm{t}} \times \tau$ pilot matrix in the $l$ th training round is denoted by $\boldsymbol{X}^{(l)}=\left[\widehat{\boldsymbol{x}}_{1}^{(l)} \ldots \widehat{\boldsymbol{x}}_{\tau}^{(l)}\right]$ for $1 \leq l \leq L$, and $\widehat{\boldsymbol{x}}_{n}^{(l)} \in \mathbb{C}^{N_{\mathrm{t}} \times 1}$ is the $n$th pilot vector, $1 \leq n \leq \tau$. It is seen that the training overhead is given by $\tau L .^{8}$ Although the RIS phase shift vector $\boldsymbol{\mu}_{l}$ can be specifically designed for minimizing both the MSE of the subchannel estimator and the computational complexity of the LS algorithm, the training overhead cannot be reduced by simply changing the RIS phase shifts. The innovative method discussed in [25] can be applied to alleviate the overhead by grouping the RIS elements and adopting a common phase shift in each group, which is detailed in Section III-C for

\footnotetext{
${ }^{7}$ Although it is somewhat surprising, the rank-deficient nature of the subchannels does not affect the estimation process. It is observed from (10) that the estimation method is feasible only if $\boldsymbol{\Sigma}$ has full row rank.

${ }^{8}$ The training overhead of $\tau L$ is the same as that of the tensor based techniques proposed in [36], and it is also equal to that of the method developed in [27], whereby the cascaded channels of every receiver antenna are separately estimated.
} 


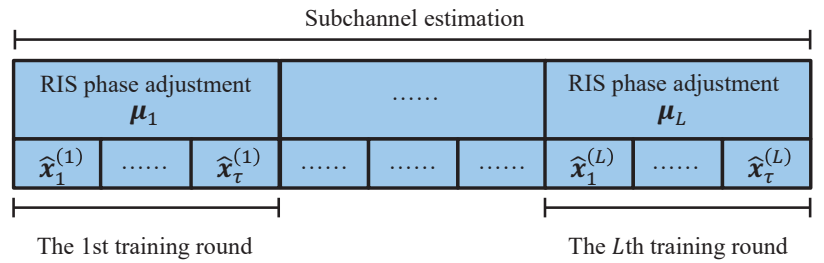

Fig. 2. Pilot pattern of the proposed subchannel estimation method.

the subchannel model. However, further reducing the training overhead is left for our future research.

\section{B. Optimal Phase Shift Design for MSE Minimization}

Based on (12), given that $\widetilde{Z}_{l}, 1 \leq l \leq L$, is i.i.d., the MSE of the LS estimator can hence be reformulated as

$$
\operatorname{MSE}\left(\hat{\boldsymbol{H}}_{s}\right)=\left\|\left(\left(\boldsymbol{\Sigma} \boldsymbol{\Sigma}^{\mathrm{H}}\right)^{-1} \boldsymbol{\Sigma}\right)\right\|_{F}^{2} \cdot \mathrm{E}\left(\left\|\widetilde{\boldsymbol{Z}}_{1}\right\|_{F}^{2}\right) .
$$

Observing that $\mathrm{E}\left(\left\|\widetilde{\boldsymbol{Z}}_{1}\right\|_{F}^{2}\right)$ is decoupled from $\boldsymbol{\Sigma}$, we have

$$
\left\|\left(\left(\boldsymbol{\Sigma} \boldsymbol{\Sigma}^{\mathrm{H}}\right)^{-1} \boldsymbol{\Sigma}\right)\right\|_{F}^{2}=\operatorname{tr}\left(\left(\boldsymbol{\Sigma} \boldsymbol{\Sigma}^{\mathrm{H}}\right)^{-1}\right)=\sum_{m=1}^{M+1} \frac{1}{\sigma_{m}^{2}(\boldsymbol{\Sigma})},
$$

where $\sigma_{m}(\boldsymbol{\Sigma})$ is the $m$ th largest singular value of $\boldsymbol{\Sigma}$. The problem of MSE minimization can thus be formulated as follows

$$
\begin{aligned}
(\mathrm{P} 1): \boldsymbol{\Sigma}^{\mathrm{opt}}= & \arg \min _{\boldsymbol{\Sigma} \in \mathbb{C}^{(M+1) \times L}} \sum_{m=1}^{M+1} \frac{1}{\sigma_{m}^{2}(\boldsymbol{\Sigma})} \\
\text { s.t. } \quad & {[\boldsymbol{\Sigma}]_{(1,:)}=\mathbf{1}_{L}, } \\
& \left|[\boldsymbol{\Sigma}]_{i, j}\right|=1, \quad \forall i, j, \\
& \operatorname{rank}\left(\boldsymbol{\Sigma} \boldsymbol{\Sigma}^{\mathrm{H}}\right)=M+1 .
\end{aligned}
$$

Prior to solving (P1), we present a sufficient condition for its feasibility as follows.

Proposition 1: Problem (P1) is feasible for any $L \geq M+1$.

Proof: Let us denote the feasible domain of $(\mathrm{P} 1)$ as $\mathcal{F}_{(\mathrm{P} 1)}$ for convenience, and set $\boldsymbol{\Sigma}_{0} \in \mathbb{C}^{(M+1) \times L}$ as follows

$$
\left[\boldsymbol{\Sigma}_{0}\right]_{(i, j)}=\left\{\begin{aligned}
1, & \text { if } i \leq j \\
-1, & \text { if } i>j .
\end{aligned}\right.
$$

The proof is completed by verifying that $\Sigma_{0}$ satisfies the constraints of (16)-(18).

The feasibility of (P1) verifies that the method given in (10) is indeed applicable to the estimation of the subchannels. Denoting the feasible domain of (P1) as $\mathcal{F}_{(\mathrm{P} 1)}$, we now present a sufficient condition for finding the optimal solution to (P1) as follows.

Proposition 2: The optimal value of $(\mathrm{P} 1)$ is $(M+1) / L$. Moreover, given any $(M+1) \times L$ matrix $\boldsymbol{A} \in \mathcal{F}_{(\mathrm{P} 1)}, \boldsymbol{A}$ is an optimal solution to (P1) if and only if $\boldsymbol{A} \boldsymbol{A}^{\mathrm{H}}=L \boldsymbol{I}_{M+1}$.

Proof: Note that

$$
\sum_{m=1}^{M+1} \sigma_{m}^{2}(\boldsymbol{\Sigma})=\operatorname{tr}\left(\left(\boldsymbol{\Sigma} \boldsymbol{\Sigma}^{\mathrm{H}}\right)\right)=L(M+1) .
$$

By exploiting the Cauchy-Schwarz inequality, we have

$$
\sum_{m=1}^{M+1} \frac{1}{\sigma_{m}^{2}(\boldsymbol{\Sigma})} \geq \frac{(M+1)^{2}}{\sum_{m=1}^{M+1} \sigma_{m}^{2}(\boldsymbol{\Sigma})}=\frac{M+1}{L},
$$

$\overline{\text { Algorithm } 1 \text { Subchannel estimation algorithm using the opti- }}$ mal codebook.

1: Set the iteration number $l=1$.

2: repeat

3: The transmitter configures the phase shift vector as $\overline{\boldsymbol{\mu}}_{l}=\left[\boldsymbol{A}^{*}\right]_{(:, l)}$.

4: The receiver enforces pilot training to obtain $\hat{\boldsymbol{H}}_{\mathrm{e}, l}$ according to (5).

5: $\quad$ Update $l=l+1$.

6: until $l>L$.

7: The receiver estimates the subchannels based on (10): $\left[\hat{\boldsymbol{H}}_{0}^{\mathrm{T}}, \ldots, \hat{\boldsymbol{H}}_{M}^{\mathrm{T}}\right]^{\mathrm{T}}=\frac{1}{L}\left(\boldsymbol{A} \otimes \boldsymbol{I}_{N_{\mathrm{r}}}\right)\left[\hat{\boldsymbol{H}}_{\mathrm{e}, 1}^{\mathrm{T}}, \ldots, \hat{\boldsymbol{H}}_{\mathrm{e}, L}^{\mathrm{T}}\right]^{\mathrm{T}}$.

8: The receiver feeds back the estimated subchannels to the transmitter.

where the equality holds if all the singular values are equal, i.e., $\sigma_{m}(\boldsymbol{\Sigma})=\sqrt{L}, 1 \leq m \leq M+1$. In this case, the optimal value of (P1) is obtained as $(M+1) / L$.

1) Sufficiency: For any $(M+1) \times L$ matrix $\boldsymbol{A} \in \mathcal{F}_{(\mathrm{P} 1)}$, if $\boldsymbol{A} \boldsymbol{A}^{\mathrm{H}}=L \boldsymbol{I}_{M+1}$, we have $\sigma_{m}(\boldsymbol{A})=\sqrt{L}, 1 \leq m \leq M+1$, which results in the equality in (21).

2) Necessity: For any $\boldsymbol{A} \in \mathcal{F}_{(\mathrm{P} 1)}$, if $\sigma_{m}(\boldsymbol{A})=\sqrt{L}, 1 \leq$ $m \leq M+1$, namely that all the eigenvalues of $\boldsymbol{A} \boldsymbol{A}^{\mathrm{H}}$ are equal to $L$, upon invoking the classic eigenvalue decomposition, we have $\boldsymbol{A} \boldsymbol{A}^{\mathrm{H}}=\boldsymbol{Q} L \boldsymbol{I}_{M+1} \boldsymbol{Q}^{\mathrm{H}}=L \boldsymbol{I}_{M+1}$, where $\boldsymbol{Q}$ is unitary such that $Q Q^{\mathrm{H}}=\boldsymbol{I}_{M+1}$.

Based on Proposition 2, Problem (P1) can be solved by searching for a scaled $L$-dimensional unitary matrix that satisfies the constraints of (16) and (17). This also indicates that the optimal solution of (P1) is not unique. For convenience, we provide two optimal designs by introducing the following two corollaries.

Corollary 1: Denote the $L$-dimensional DFT matrix as $\boldsymbol{F}_{L}$, i.e., $\left[\boldsymbol{F}_{L}\right]_{m, n}=e^{-\mathrm{j} \frac{2 \pi(m-1)(n-1)}{L}}$. Let $\boldsymbol{A} \in \mathbb{C}^{(M+1) \times L}$ host the first and $M$ other distinct rows of $\boldsymbol{F}_{L}$. Then $\boldsymbol{A}$ constitutes an optimal solution to (P1).

Proof: The proof is completed by verifying that $\boldsymbol{A} \in$ $\mathcal{F}_{(\mathrm{P} 1)}$ and $\boldsymbol{A} \boldsymbol{A}^{\mathrm{H}}=L \boldsymbol{I}_{M+1}$.

Before presenting the second corollary, we introduce the Hadamard matrix. Without loss of generality, let $L=2^{B}$, where $B>0$ is an integer. Then the $2^{B}$-dimensional Hadamard matrix, $\boldsymbol{G}_{2^{B}}$, can be constructed by

$$
\boldsymbol{G}_{2^{B}}=\left[\begin{array}{cc}
\boldsymbol{G}_{2^{B-1}} & \boldsymbol{G}_{2^{B-1}} \\
\boldsymbol{G}_{2^{B-1}} & -\boldsymbol{G}_{2^{B-1}}
\end{array}\right], \boldsymbol{G}_{2}=\left[\begin{array}{cc}
1 & 1 \\
1 & -1
\end{array}\right] .
$$

It can be verified that $\boldsymbol{G}_{2^{B}} \boldsymbol{G}_{2^{B}}^{\mathrm{H}}=2^{B} \boldsymbol{I}_{2^{B}}$.

Corollary 2: Let us denote the $L$-dimensional Hadamard matrix in (22) as $\boldsymbol{G}_{L}$, and let $\boldsymbol{A} \in \mathbb{C}^{(M+1) \times L}$ host the first and $M$ other distinct rows of $\boldsymbol{G}_{L}$. Then $\boldsymbol{A}$ constitutes an optimal solution to (P1).

Proof: The proof is completed by verifying that $\boldsymbol{A} \in$ $\mathcal{F}_{(\mathrm{P} 1)}$ and $\boldsymbol{A} \boldsymbol{A}^{\mathrm{H}}=L \boldsymbol{I}_{M+1}$.

In practical systems, the optimized phase adjustments provided by the RIS for subchannel estimation can be specified in advance as codebooks at the transceiver. Note that different choices of feasible codebooks do not change the training overhead, but the optimal codebooks given by Corollaries 1 
and 2 can be used to minimize the MSE of the estimator, and also to simplify the classic LS algorithm formulated in (10), by letting $\left(\boldsymbol{A} \boldsymbol{A}^{\mathrm{H}}\right)^{-1} \boldsymbol{A}=\frac{1}{L} \boldsymbol{A}$. The main procedures of the proposed subchannel estimation method are summarized in Algorithm 1.

It is worth noting that the Hadamard codebook given in Corollary 2 requires only two discrete phase shifts, namely $\{0, \pi\}$, which is appealing for practical applications. Furthermore, based on the channel decomposition model of (7), the subchannel estimation methods are not limited to the typical LS algorithm. Indeed, many practical algorithms, such as the minimum mean square error (MMSE) [27], the linear MMSE (LMMSE) [37], or any other new solutions, can also be applied. Instead of estimating the subchannels using the effective channels of (8) observed in each training round, one can also consider to jointly process all the received pilots $\boldsymbol{Y}_{l}$, $1 \leq l \leq L$, to acquire the CSI of the subchannels, where $\boldsymbol{Y}_{l} \in \mathbb{C}^{N_{\mathrm{r}} \times \tau}$ is the signal received in the $l$ th round of training. Since the same phase shifts are imposed on the incident signal across the whole transmission bandwidth, the proposed method can be readily extended to the case of frequency-selective fading channels by processing the subchannels of different subcarriers independently.

\section{Performance Analysis}

1) General performance of the subchannel estimator: Given an optimal codebook $\boldsymbol{A} \in \mathcal{F}_{(\mathrm{P} 1)}$ that satisfies $\boldsymbol{A} \boldsymbol{A}^{\mathrm{H}}=$ $L \boldsymbol{I}_{M+1}$, the $(M+1) N_{\mathrm{r}} \times N_{\mathrm{t}}$ subchannel estimator is formulated as

$$
\begin{aligned}
\hat{\boldsymbol{H}}_{s}^{\mathrm{opt}} & =\frac{1}{L}\left(\boldsymbol{A} \otimes \boldsymbol{I}_{N_{\mathrm{r}}}\right)\left[\hat{\boldsymbol{H}}_{\mathrm{e}, 1}^{\mathrm{T}}, \ldots, \hat{\boldsymbol{H}}_{\mathrm{e}, L}^{\mathrm{T}}\right]^{\mathrm{T}} \\
& =\boldsymbol{H}_{s}+\frac{1}{L}\left(\boldsymbol{A} \otimes \boldsymbol{I}_{N_{\mathrm{r}}}\right) \widetilde{\boldsymbol{\Xi}},
\end{aligned}
$$

and the MSE is given by $\operatorname{MSE}\left(\hat{\boldsymbol{H}}_{s}^{\text {opt }}\right)=\frac{M+1}{L} \mathrm{E}\left(\left\|\widetilde{\boldsymbol{Z}}_{1}\right\|_{F}^{2}\right)$, where $\widetilde{Z}_{1}$ is the effective noise of (5).

Before summarizing the general performance of the subchannel estimator in Proposition 3, we first present a lemma as follows.

Lemma 1: The $(M+1) N_{\mathrm{r}} N_{\mathrm{t}}$-dimensional covariance matrix that attains the Cramer-Rao lower bound (CRLB) of the subchannel estimator derived from (9) is given by

$$
\boldsymbol{\Theta}_{\mathrm{CRLB}}=\sigma^{2}\left(\boldsymbol{X}^{*} \boldsymbol{X}^{\mathrm{T}}\right)^{-1} \otimes\left(\boldsymbol{A} \boldsymbol{A}^{\mathrm{H}}\right)^{-1} \otimes \boldsymbol{I}_{N_{\mathrm{r}}}
$$

Proof: See Appendix A for the proof.

Proposition 3: The subchannel estimator in (23) is unbiased, consistent, and efficient.

Proof: The unbiased nature is validated by $\mathrm{E}(\widetilde{\boldsymbol{\Xi}})=$ $\left[\mathrm{E}\left(\widetilde{\boldsymbol{Z}}_{1}^{\mathrm{T}}\right), \ldots, \mathrm{E}\left(\widetilde{\boldsymbol{Z}}_{L}^{\mathrm{T}}\right)\right]^{\mathrm{T}}=\mathbf{0}$. Additionally, the power of the effective noise is bounded by

$$
\mathrm{E}\left(\left\|\widetilde{\boldsymbol{Z}}_{1}\right\|_{F}^{2}\right)=\sum_{n=1}^{N_{\mathrm{t}}} \frac{\sigma^{2} N_{\mathrm{r}}}{\sigma_{n}^{2}(\boldsymbol{X})} \leq \frac{\sigma^{2} N_{\mathrm{r}} N_{\mathrm{t}}}{\lambda_{N_{\mathrm{t}}}(\boldsymbol{X})}
$$

where $\sigma_{n}(\boldsymbol{X})$ and $\lambda_{n}(\boldsymbol{X})$ are the $n$th largest singular value and eigenvalue of the pilot matrix $\boldsymbol{X}$, respectively. Therefore, the consistency is proved by

$$
\lim _{L \rightarrow \infty} \operatorname{MSE}\left(\hat{\boldsymbol{H}}_{s}^{\mathrm{opt}}\right) \leq \lim _{L \rightarrow \infty} \frac{\frac{(M+1) \sigma^{2} N_{\mathrm{r}} N_{\mathrm{t}}}{\lambda_{N_{\mathrm{t}}}(\boldsymbol{X})}}{L}=0 .
$$

Moreover, based on Lemma 1, we have

$$
\operatorname{MSE}\left(\hat{\boldsymbol{H}}_{s}^{\mathrm{opt}}\right)=\frac{M+1}{L} \sum_{n=1}^{N_{\mathrm{t}}} \frac{\sigma^{2} N_{\mathrm{r}}}{\sigma_{n}^{2}(\boldsymbol{X})}=\operatorname{tr}\left(\boldsymbol{\Theta}_{\mathrm{CRLB}}\right),
$$

which thus verifies the efficiency.

2) Complexity analysis: The computational complexity is mainly contributed by the $L$ rounds of pilot training in (5) and the subchannel estimation in (10). It is easy to verify that the complexity of a single round of pilot training is on the order of $\mathcal{O}\left(\tau N_{\mathrm{t}}^{2}\right)$. As for subchannel estimation, relying on an optimal codebook $\boldsymbol{A}$, the complexity is dominated by the computation of $\frac{1}{L}\left(\boldsymbol{A} \otimes \boldsymbol{I}_{N_{\mathrm{r}}}\right)\left[\hat{\boldsymbol{H}}_{\mathrm{e}, 1}^{\mathrm{T}}, \ldots, \hat{\boldsymbol{H}}_{\mathrm{e}, L}^{\mathrm{T}}\right]^{\mathrm{T}}$. The matrix multiplication can be considered as an augmented version of $\frac{1}{L} \boldsymbol{A} \boldsymbol{h}$ with $\boldsymbol{h}$ denoting an $L \times 1$ vector. Thus, the complexity of subchannel estimation is $N_{\mathrm{r}} N_{\mathrm{t}}$ times that of computing $\frac{1}{L} \boldsymbol{A} \boldsymbol{h}$, which is given by $\mathcal{O}\left(N_{\mathrm{r}} N_{\mathrm{t}} L M\right)$. Therefore, the total complexity is $\mathcal{O}\left(N_{\mathrm{t}} L\left(N_{\mathrm{r}} M+\tau N_{\mathrm{t}}\right)\right)$.

Naturally, numerous techniques may be applied to reduce the computational complexity of subchannel estimation. Without loss of generality, let $L=M+1=2^{B}$. Then for the DFT codebook, i.e., $\boldsymbol{A}=\boldsymbol{F}_{M+1}$, the complexity of computing $\frac{1}{L} \boldsymbol{F}_{M+1} \boldsymbol{h}$ is reduced to $\mathcal{O}(M \log M)$ by the fast Fourier transform (FFT) operations. As for the case of using the Hadamard codebook in (22), let us denote the complexity of computing $\frac{1}{L} \boldsymbol{G}_{2^{B}} \boldsymbol{h}$ by $T(B)$. Then the update formula of $T(B)$ is given by

$$
T(B)=2 T(B-1)+2^{B} .
$$

Thus, we have $T(B)=\mathcal{O}\left(B 2^{B}\right)=\mathcal{O}(M \log M)$. The total complexity is then reduced to $\mathcal{O}\left(N_{\mathrm{t}} M\left(N_{\mathrm{r}} \log M+\tau N_{\mathrm{t}}\right)\right)$.

The proposed subchannel estimation algorithm can be further simplified by collecting several subchannels into groups with a group-specific common phase shift. Let us denote the index set of the $g$ th subchannel group as $\mathcal{S}_{g}, 1 \leq g \leq N_{\mathrm{g}}$, and assume that the subchannels are divided into $N_{\mathrm{g}}\left(N_{\mathrm{g}} \leq M+1\right)$ groups by letting $\cup_{g=1}^{N_{\mathrm{g}}} \mathcal{S}_{g}=\{0, \cdots, M\}$ and $\mathcal{S}_{g_{1}} \cap \mathcal{S}_{g_{2}}=\varnothing$ for $\forall g_{1} \neq g_{2}$. Then the phase shift vector $\widetilde{\boldsymbol{\mu}}_{g}$ is shared among all the subchannels in the $g$ th group. Based on this, the decomposed effective channel in (6) is reformulated as

$$
\boldsymbol{H}_{\mathrm{e}}(\boldsymbol{\mu})=\sum_{g=1}^{N_{\mathrm{g}}} \widetilde{\boldsymbol{\mu}}_{g} \boldsymbol{H}_{g}^{\mathrm{sup}}
$$

where $\boldsymbol{H}_{g}^{\text {sup }}=\sum_{m \in \mathcal{S}_{g}} \boldsymbol{H}_{m}$ is the $g$ th superposed channel. In this manner, the size of the codebook $\boldsymbol{A}$ is reduced from $(M+1) \times L$ to $N_{\mathrm{g}} \times L$, which has a lower complexity of $\mathcal{O}\left(N_{\mathrm{t}} N_{\mathrm{g}}\left(N_{\mathrm{r}} \log N_{\mathrm{g}}+\tau N_{\mathrm{t}}\right)\right)$ if we have $L=N_{\mathrm{g}}$ for simplicity. ${ }^{9}$ Observe that for a smaller value of $N_{\mathrm{g}}$, the estimation algorithm becomes less complex. Given $N_{\mathrm{g}}=1$, the computational complexity can even be reduced to $\mathcal{O}\left(\tau N_{\mathrm{t}}^{2}\right)$.

\footnotetext{
${ }^{9}$ By grouping the subchannels, the minimum value of $L$ is reduced to the number of the groups $N_{\mathrm{g}}$, instead of $M+1$. Hence, the complexity of the estimation process can be much reduced.
} 
However, the CSI resolution also becomes lower when only relying on the knowledge of the superposed channels. Hence, the value of $N_{\mathrm{g}}$ should be carefully chosen to strike an appealing CSI precision versus complexity trade-off. Finally, the estimation of subchannels can be further simplified using low-complexity pilot training methods.

In this section, we have shown that the CSI of the subchannels can be acquired. Since the existing RIS-aided precoding designs tend to rely on the idealized assumption of perfect CSI, we design a new precoder purely relying on the knowledge of the subchannels in the next section. The feasibility of the precoding design of Section IV is guaranteed by the subchannel estimation method of Section III.

\section{Proposed Precoding Design Relying on the CSI OF SUBCHANNELS}

In this section, we deal with the precoding problem purely relying on the knowledge of the subchannels $\boldsymbol{H}_{m}$, rather than on the full CSI of $\boldsymbol{H}_{\mathrm{t}}$ and $\boldsymbol{H}_{\mathrm{r}} \cdot{ }^{10}$ To circumvent the challenge of finding the closed-form optimal design of the phase shift vector and the digital precoder, we conceive a near-optimal two-stage precoding scheme. Firstly, the phase shift vector is determined using a fixed-point based method, based on which the digital precoder may then be derived by solving a convex optimization problem. Then, considering that the performance gap between the proposed design and the optimal precoding solution is analytically intractable, we investigate a simple scenario and show that the proposed design is capable of attaining the optimal performance for the propagation conditions considered, where the RIS can act as though both the transmission power and the number of TAs were increased by exploiting the reflected link.

\section{A. Precoding Framework with CSI of Subchannels}

With the channel decomposition model of (7), the received signal of (1) is reformulated as

$$
\boldsymbol{y}=\left[\boldsymbol{H}_{0}, \ldots, \boldsymbol{H}_{M}\right](\overline{\boldsymbol{\mu}} \otimes \boldsymbol{W}) \boldsymbol{s}+\boldsymbol{z} .
$$

The effective mutual information (MI) achieved by Gaussian signaling can be adopted as the optimization objective of our precoding design [38]-[41], which is expressed as

$$
I(\overline{\boldsymbol{\mu}}, \boldsymbol{W})=\log _{2}\left|\boldsymbol{I}_{N_{\mathrm{r}}}+\frac{\rho}{\sigma^{2}} \boldsymbol{H}_{\mathrm{eq}}(\overline{\boldsymbol{\mu}} \otimes \boldsymbol{W})(\overline{\boldsymbol{\mu}} \otimes \boldsymbol{W})^{\mathrm{H}} \boldsymbol{H}_{\mathrm{eq}}^{\mathrm{H}}\right|,
$$

where $\boldsymbol{H}_{\mathrm{eq}}=\left[\boldsymbol{H}_{0}, \ldots, \boldsymbol{H}_{M}\right]$. Upon using the MI metric, no common phase rotation applied to the entries of $\overline{\boldsymbol{\mu}}$ changes the objective, and the joint precoding design may then be for-

\footnotetext{
${ }^{10}$ Again, the existing precoders rely on the full CSI of $\boldsymbol{H}_{\mathrm{t}}$ and $\boldsymbol{H}_{\mathrm{r}}$, and hence cannot be directly applied when only the knowledge of the subchannels is available.
}

mulated as the following constrained optimization problem ${ }^{11}$

$$
\begin{aligned}
(\mathrm{P} 2): \quad\left(\overline{\boldsymbol{\mu}}^{\mathrm{opt}}, \boldsymbol{W}^{\mathrm{opt}}\right)= & \arg \max _{\overline{\boldsymbol{\mu}}, \boldsymbol{W}} I(\overline{\boldsymbol{\mu}}, \boldsymbol{W}) \\
\text { s.t. } & \left|[\overline{\boldsymbol{\mu}}]_{m}\right|=1, \quad \forall m, \\
& \|\boldsymbol{W}\|_{F}^{2} \leq 1 .
\end{aligned}
$$

Note that Problem (P2) is difficult to solve both owing to the non-convex constraint of (33) and due to the Kronecker product structure of $\overline{\boldsymbol{\mu}} \otimes \boldsymbol{W}$. Fortunately, it is seen that for a fixed $\overline{\boldsymbol{\mu}}$, the design of $\boldsymbol{W}$ that maximizes the MI becomes a convex problem. Thus, it is efficient to solve (P2) by conceiving a suboptimal two-stage scheme that successively determines $\overline{\boldsymbol{\mu}}$ and $\boldsymbol{W}$.

In the first stage, in order to find a near-optimal design of $\overline{\boldsymbol{\mu}}$, we develop a lower bound of $I(\overline{\boldsymbol{\mu}}, \boldsymbol{W})$, and obtain $\overline{\boldsymbol{\mu}}$ by maximizing this lower bound. The bound can be derived based on the following proposition.

Proposition 4: Define $\mathcal{F}_{\boldsymbol{P}} \triangleq\left\{\boldsymbol{P} \in \mathbb{C}^{(M+1) N_{\mathrm{t}} \times N_{\mathrm{s}}}\right.$ : $\left.\|\boldsymbol{P}\|_{F}^{2} \leq M+1\right\}$, and $\tilde{I}(\boldsymbol{P})=\log _{2}\left|\boldsymbol{I}_{N_{\mathrm{r}}}+\frac{\rho}{\sigma^{2}} \boldsymbol{H}_{\mathrm{eq}} \boldsymbol{P} \boldsymbol{P}^{\mathrm{H}} \boldsymbol{H}_{\mathrm{eq}}^{\mathrm{H}}\right|$. Let $\boldsymbol{P}^{\text {opt }}=\arg \max _{\boldsymbol{P} \in \mathcal{F}_{P}} \tilde{I}(\boldsymbol{P})$. Then for any feasible $(\overline{\boldsymbol{\mu}}, \boldsymbol{W})$, the gap between the objective of (P2) and $\tilde{I}\left(\boldsymbol{P}^{\mathrm{opt}}\right)$ is bounded by

$$
\left|I(\overline{\boldsymbol{\mu}}, \boldsymbol{W})-\tilde{I}\left(\boldsymbol{P}^{\mathrm{opt}}\right)\right| \leq C\left\|\overline{\boldsymbol{\mu}} \otimes \boldsymbol{W}-\boldsymbol{P}^{\mathrm{opt}}\right\|_{F},
$$

where $C \in \mathbb{R}$ is a non-negative constant.

Proof: See Appendix B for the proof.

Observe that $\mathcal{F}_{\boldsymbol{P}}$ is a convex set, and thus $\boldsymbol{P}^{\text {opt }}$ that maximizes $\tilde{I}(\boldsymbol{P})$ is analytically attainable [41]. Specifically, let the singular value decomposition (SVD) of $\boldsymbol{H}_{\text {eq }}$ be $\boldsymbol{H}_{\mathrm{eq}}=\boldsymbol{U} \boldsymbol{S} \boldsymbol{V}^{\mathrm{H}}$, where $\boldsymbol{S}$ is a $\operatorname{rank}\left(\boldsymbol{H}_{\mathrm{eq}}\right) \times \operatorname{rank}\left(\boldsymbol{H}_{\mathrm{eq}}\right)$ diagonal matrix of singular values arranged in descending order, while $\boldsymbol{U} \in \mathbb{C}^{N_{\mathrm{r}} \times \operatorname{rank}\left(\boldsymbol{H}_{\text {eq }}\right)}, \boldsymbol{U}^{\mathrm{H}} \boldsymbol{U}=\boldsymbol{I}_{\mathrm{rank}\left(\boldsymbol{H}_{\text {eq }}\right)}$, and $\boldsymbol{V} \in \mathbb{C}^{(M+1) N_{\mathrm{t}} \times \operatorname{rank}\left(\boldsymbol{H}_{\mathrm{eq}}\right)}, \boldsymbol{V}^{\mathrm{H}} \boldsymbol{V}=\boldsymbol{I}_{\operatorname{rank}\left(\boldsymbol{H}_{\mathrm{eq}}\right)}$. Without loss of generality, we assume $N_{\mathrm{s}} \leq \operatorname{rank}\left(\boldsymbol{H}_{\mathrm{eq}}\right)$. Then $\boldsymbol{P}^{\text {opt }}$ is derived from the first $N_{\mathrm{s}}$ columns of $\boldsymbol{V}$, i.e., $\boldsymbol{P}^{\text {opt }}=$ $\sqrt{(M+1) / N_{\mathrm{s}}}[\boldsymbol{V}]_{\left(:, 1: N_{\mathrm{s}}\right)}$.

It is worth noting that $\tilde{I}\left(\boldsymbol{P}^{\mathrm{opt}}\right)$ serves as an upper bound for the optimal value of $(\mathrm{P} 2)$, i.e., $\tilde{I}\left(\boldsymbol{P}^{\mathrm{opt}}\right) \geq I\left(\overline{\boldsymbol{\mu}}^{\mathrm{opt}}, \boldsymbol{W}^{\mathrm{opt}}\right) .^{12}$ However, in most cases, this bound is not approachable, because $\boldsymbol{P}^{\text {opt }}$ cannot be decomposed into a Kronecker product of a vector with unit-magnitude entries and an $F$-normconstrained complex matrix. Therefore, we derive a lower bound of the MI to assist with the suboptimal design of $\bar{\mu}$.

Based on Proposition 4, a lower bound of $I(\overline{\boldsymbol{\mu}}, \boldsymbol{W})$ can be formulated as follows

$$
\begin{aligned}
I(\overline{\boldsymbol{\mu}}, \boldsymbol{W}) & \geq \tilde{I}\left(\boldsymbol{P}^{\mathrm{opt}}\right)-C\left\|\overline{\boldsymbol{\mu}} \otimes \boldsymbol{W}-\boldsymbol{P}^{\mathrm{opt}}\right\|_{F} \\
& \geq \underbrace{I\left(\overline{\boldsymbol{\mu}}^{\mathrm{opt}}, \boldsymbol{W}^{\mathrm{opt}}\right)-C\left\|\overline{\boldsymbol{\mu}} \otimes \boldsymbol{W}-\boldsymbol{P}^{\mathrm{opt}}\right\|_{F}}_{I_{\mathrm{LB}}, \text { the lower bound of } I(\overline{\boldsymbol{\mu}}, \boldsymbol{W})} .
\end{aligned}
$$

\footnotetext{
${ }^{11}$ For fast changing channels associated with limited coherence intervals, pilot-based channel estimation will become impractical. More explicitly, every time the Doppler frequency is doubled, the pilot overhead used for sampling and estimating the channel has to be doubled as well. In this scenario, it is worth considering to maximize the ergodic MI, $\mathrm{E}\{I(\overline{\boldsymbol{\mu}}, \boldsymbol{W})\}$, instead of the instantaneous MI, $I(\overline{\boldsymbol{\mu}}, \boldsymbol{W})$, so that only the statistical properties of the channels are required..

${ }^{12}$ This is due to the fact that $\left(\overline{\boldsymbol{\mu}}^{\text {opt }} \otimes \boldsymbol{W}^{\text {opt }}\right) \in \mathbb{C}^{(M+1) N_{\mathrm{t}} \times N_{\mathrm{s}}}$ and $\left\|\overline{\boldsymbol{\mu}}^{\mathrm{opt}} \otimes \boldsymbol{W}^{\mathrm{opt}}\right\|_{F}^{2} \leq M+1$.
} 
This implies that $I(\overline{\boldsymbol{\mu}}, \boldsymbol{W})$ can be approximately maximized by maximizing its lower bound $I_{\mathrm{LB}}$, or equivalently minimizing $\left\|\overline{\boldsymbol{\mu}} \otimes \boldsymbol{W}-\boldsymbol{P}^{\mathrm{opt}}\right\|_{F}$, and thus the phase shift vector of the two-stage scheme $\overline{\boldsymbol{\mu}}^{(\mathrm{ts})}$ can be derived by solving the following problem ${ }^{13}$

$$
\begin{gathered}
\text { (P3) : } \quad\left(\overline{\boldsymbol{\mu}}^{(\mathrm{ts})}, \sim\right)=\arg \min _{\overline{\boldsymbol{\mu}}, \boldsymbol{W}}\left\|\overline{\boldsymbol{\mu}} \otimes \boldsymbol{W}-\boldsymbol{P}^{\mathrm{opt}}\right\|_{F}^{2} \\
\text { s.t. (33)-(34). }
\end{gathered}
$$

Based on $\overline{\boldsymbol{\mu}}^{(\mathrm{ts})}$ that solves (P3), the digital precoder $\boldsymbol{W}^{(\mathrm{ts})}$ is determined by maximizing $I\left(\overline{\boldsymbol{\mu}}^{(\mathrm{ts})}, \boldsymbol{W}\right)$, which yields the following optimization problem

$$
\begin{gathered}
(\mathrm{P} 4): \quad \boldsymbol{W}^{(\mathrm{ts})}=\arg \max _{\boldsymbol{W}} I\left(\overline{\boldsymbol{\mu}}^{(\mathrm{ts})}, \boldsymbol{W}\right) \\
\text { s.t. }\|\boldsymbol{W}\|_{F}^{2} \leq 1 .
\end{gathered}
$$

The joint precoding solution derived from the two-stage scheme is then given by $\left(\overline{\boldsymbol{\mu}}^{(\mathrm{ts})}, \boldsymbol{W}^{(\mathrm{ts})}\right)$.

\section{B. Two-stage Precoding Designs}

1) Fixed-point based method for solving (P3): Due to the non-convex constraints of (P3), it is unrealistic to search for the closed-form optimal solution. Hence, in order to solve (P3) efficiently, a fixed-point based method is developed to determine the phase shift vector $\overline{\boldsymbol{\mu}}^{(\mathrm{ts})}$.

For convenience, let us introduce $\boldsymbol{P}^{\mathrm{opt}}=\left[\boldsymbol{P}_{0}^{\mathrm{T}}, \ldots, \boldsymbol{P}_{M}^{\mathrm{T}}\right]^{\mathrm{T}}$, where $\boldsymbol{P}_{m} \in \mathbb{C}^{N_{\mathrm{t}} \times N_{\mathrm{s}}}, 1 \leq m \leq M+1$, is the corresponding submatrix of $\boldsymbol{P}^{\text {opt }}$. Then we have

$$
\begin{gathered}
\left\|\overline{\boldsymbol{\mu}} \otimes \boldsymbol{W}-\boldsymbol{P}^{\mathrm{opt}}\right\|_{F}^{2}=\sum_{m=1}^{M+1}\left\|\boldsymbol{W}-\bar{\mu}_{m}^{*} \boldsymbol{P}_{m}\right\|_{F}^{2} \\
=(M+1)\left\|\boldsymbol{W}-\sum_{m=1}^{M+1} \frac{\bar{\mu}_{m}^{*} \boldsymbol{P}_{m}}{M+1}\right\|_{F}^{2} \\
\quad+\sum_{m=1}^{M+1}\left\|\boldsymbol{P}_{m}\right\|_{F}^{2}-\frac{1}{M+1}\left\|\sum_{m=1}^{M+1} \bar{\mu}_{m}^{*} \boldsymbol{P}_{m}\right\|_{F}^{2} \\
\geq \sum_{m=1}^{M+1}\left\|\boldsymbol{P}_{m}\right\|_{F}^{2}-\frac{1}{M+1}\left\|\sum_{m=1}^{M+1} \bar{\mu}_{m}^{*} \boldsymbol{P}_{m}\right\|_{F}^{2},
\end{gathered}
$$

where $\bar{\mu}_{m}=[\overline{\boldsymbol{\mu}}]_{m}$ is the $m$ th entry of the phase shift vector, and the equality holds if $\boldsymbol{W}=\sum_{m=1}^{M+1} \frac{\bar{\mu}_{m}^{*} \boldsymbol{P}_{m}}{M+1}$. It is noted that $\left\|\sum_{m=1}^{M+1} \frac{\bar{\mu}_{m}^{*} \boldsymbol{P}_{m}}{M+1}\right\|_{F}^{2} \leq(M+1) \sum_{m=1}^{M+1}\left\|\frac{\bar{\mu}_{m}^{*} \boldsymbol{P}_{m}}{M+1}\right\|_{F}^{2}=1$, and thus this design of the digital precoder is feasible. Hence, minimizing the objective of (P3) is then equivalent to maximizing $\left\|\sum_{m=1}^{M+1} \bar{\mu}_{m}^{*} \boldsymbol{P}_{m}\right\|_{F}^{2}$. Furthermore, we have

$$
\begin{gathered}
\left\|\sum_{m=1}^{M+1} \bar{\mu}_{m}^{*} \boldsymbol{P}_{m}\right\|_{F}^{2}=\operatorname{tr}\left[\left(\sum_{n=1}^{M+1} \bar{\mu}_{n}^{*} \boldsymbol{P}_{n}\right)^{\mathrm{H}}\left(\sum_{m=1}^{M+1} \bar{\mu}_{m}^{*} \boldsymbol{P}_{m}\right)\right] \\
=\sum_{n=1}^{M+1} \sum_{m=1}^{M+1} \bar{\mu}_{m}^{*} \bar{\mu}_{n} \operatorname{tr}\left(\boldsymbol{P}_{n}^{\mathrm{H}} \boldsymbol{P}_{m}\right)=\overline{\boldsymbol{\mu}}^{\mathrm{H}} \boldsymbol{Q} \overline{\boldsymbol{\mu}},
\end{gathered}
$$

\footnotetext{
${ }^{13}$ It is noted that a design of the precoding matrix, $\boldsymbol{W}^{(\mathrm{P} 3)}$, can also be obtained by solving (P3). However, given $\overline{\boldsymbol{\mu}}^{(\mathrm{ts})}$, this $\boldsymbol{W}^{(\mathrm{P} 3)}$ only maximizes the lower bound $I_{\mathrm{LB}}$ rather than the MI $I(\overline{\boldsymbol{\mu}}, \boldsymbol{W})$ itself. Hence, another optimization will be formulated in the second stage to redesign $\boldsymbol{W}$ for further increasing $I(\overline{\boldsymbol{\mu}}, \boldsymbol{W})$.
}

where $\boldsymbol{Q}$ is an $(M+1) \times(M+1)$ Hermitian matrix with entries of $[\boldsymbol{Q}]_{(m, n)}=q_{m n}=\operatorname{tr}\left(\boldsymbol{P}_{n}^{\mathrm{H}} \boldsymbol{P}_{m}\right)$. Thus, (P3) can be reformulated as

$$
\begin{array}{r}
\text { (P5) : } \quad \overline{\boldsymbol{\mu}}^{(\mathrm{ts})}=\arg \max _{\overline{\boldsymbol{\mu}}} \overline{\boldsymbol{\mu}}^{\mathrm{H}} \boldsymbol{Q} \overline{\boldsymbol{\mu}} \\
\text { s.t. }\left|[\overline{\boldsymbol{\mu}}]_{m}\right|=1, \forall m .
\end{array}
$$

Following from some general algebraic manipulations, the Karush-Kuhn-Tucker (KKT) condition of (P5) can be derived as

$$
(Q-\operatorname{diag}(\bar{\nu})) \bar{\mu}=\mathbf{0},
$$

where $\bar{\nu} \in \mathbb{R}^{(M+1) \times 1}$ is the Lagrangian multiplier. Given the great challenge of providing a closed-form solution, we develop a fixed-point based method to solve (P5) iteratively. By rearranging (42), given $\overline{\boldsymbol{\mu}}^{(p)}$ with $p$ denoting the iteration number, $\overline{\boldsymbol{\nu}}^{(p+1)}$ and $\overline{\boldsymbol{\mu}}^{(p+1)}$ can be updated as follows

$$
\overline{\boldsymbol{\mu}}^{(p+1)}=e^{\mathrm{j} \angle\left(\boldsymbol{Q} \overline{\boldsymbol{\mu}}^{(p)}\right)}, \quad \overline{\boldsymbol{\nu}}^{(p+1)}=\left|\boldsymbol{Q} \overline{\boldsymbol{\mu}}^{(p)}\right| .
$$

The residual in the $p$ th round of iterations is computed by $\delta^{(p)}=\left|\left\|\boldsymbol{Q} \overline{\boldsymbol{\mu}}^{(p+1)}\right\|_{1}-\left\|\boldsymbol{Q} \overline{\boldsymbol{\mu}}^{(p)}\right\|_{1}\right|$. By appropriately choosing the residual threshold $\epsilon$, and the maximum number of iteration $p_{\max }$, the stopping criterion can be developed based on $\delta^{(p)}<\epsilon$ or $p>p_{\max }$. The solution to (P5) is then obtained by our fixedpoint iterative algorithm.

2) SVD based method for solving (P4): Given $\overline{\boldsymbol{\mu}}^{(\mathrm{ts})}$ as the solution to (P5), the objective of (P4) is reformulated as $I\left(\overline{\boldsymbol{\mu}}^{(\mathrm{ts})}, \boldsymbol{W}\right)=\log _{2}\left|\boldsymbol{I}_{N_{\mathrm{r}}}+\frac{\rho}{\sigma^{2}} \hat{\boldsymbol{H}}_{\mathrm{eq}} \boldsymbol{W} \boldsymbol{W}^{\mathrm{H}} \hat{\boldsymbol{H}}_{\mathrm{eq}}^{\mathrm{H}}\right|$, where $\hat{\boldsymbol{H}}_{\mathrm{eq}}=\boldsymbol{H}_{\mathrm{eq}}\left(\overline{\boldsymbol{\mu}}^{(\mathrm{ts})} \otimes \boldsymbol{I}_{N_{\mathrm{t}}}\right)$. Thus, (P4) becomes a convex optimization problem, and can be solved using the same method adopted for $\boldsymbol{P}^{\mathrm{opt}}$. Specifically, let the eigenvalue decomposition of $\hat{\boldsymbol{H}}_{\mathrm{eq}}^{\mathrm{H}} \hat{\boldsymbol{H}}_{\mathrm{eq}}$ be $\hat{\boldsymbol{H}}_{\mathrm{eq}}^{\mathrm{H}} \hat{\boldsymbol{H}}_{\mathrm{eq}}=\hat{\boldsymbol{V}} \boldsymbol{\Lambda} \hat{\boldsymbol{V}}^{\mathrm{H}}$, where $\boldsymbol{\Lambda} \in \mathbb{C}^{N_{\mathrm{t}} \times N_{\mathrm{t}}}$ is a diagonal matrix of eigenvalues arranged in descending order, and $\hat{\boldsymbol{V}} \in \mathbb{C}^{N_{\mathrm{t}} \times N_{\mathrm{t}}}$ is a unitary matrix of the corresponding eigenvectors. Then $\boldsymbol{W}$ is derived from the first $N_{\mathrm{s}}$ columns of $\hat{\boldsymbol{V}}$ as follows

$$
\boldsymbol{W}^{(\mathrm{ts})}=\sqrt{1 / N_{\mathrm{s}}}[\hat{\boldsymbol{V}}]_{\left(:, 1: N_{\mathrm{s}}\right)} .
$$

In summary, the joint precoding problem is solved by determining the phase shift vector $\bar{\mu}$ using the fixed-point based iterations, and then formulating the digital precoder $W$ according to (44). The details of the proposed precoding method are presented in Algorithm 2.

\section{Performance of the Fixed-Point Based Method}

The convergence and optimality are confirmed by in the following proposition.

Proposition 5: Given $\left\{\overline{\boldsymbol{\mu}}^{(p)}\right\}$ as the output sequence of the phase shift vectors derived from Algorithm 2, then the sequence $\left\{\overline{\boldsymbol{\mu}}^{(p)}\right\}$ is convergent, and has a limit that satisfies the KKT condition of (42).

Proof: Note that the sequence $\left\{\left\|\boldsymbol{Q} \overline{\boldsymbol{\mu}}^{(p)}\right\|_{1}\right\}$ is convergent. Specifically, the sequence is bounded by $\left\|\boldsymbol{Q} \overline{\boldsymbol{\mu}}^{(p)}\right\|_{1} \leq$ $\sum_{m} \sum_{n}\left|q_{m n}\right|$. In addition, we have

$$
\begin{aligned}
\left\|\boldsymbol{Q} \overline{\boldsymbol{\mu}}^{(p)}\right\|_{1} & =\left(e^{\mathrm{j} \angle\left(\boldsymbol{Q} \overline{\boldsymbol{\mu}}^{(p)}\right)}\right)^{\mathrm{H}} \boldsymbol{Q} \overline{\boldsymbol{\mu}}^{(p)} \\
& =\left(\boldsymbol{Q} \overline{\boldsymbol{\mu}}^{(p+1)}\right)^{\mathrm{H}} \overline{\boldsymbol{\mu}}^{(p)} \leq\left\|\boldsymbol{Q} \overline{\boldsymbol{\mu}}^{(p+1)}\right\|_{1},
\end{aligned}
$$

where the inequality follows from $\left|\left[\overline{\boldsymbol{\mu}}^{(p)}\right]_{m}\right|=1$, and $\boldsymbol{Q}=$ $\boldsymbol{Q}^{\mathrm{H}}$. Thus, the convergence of $\left\{\overline{\boldsymbol{\mu}}^{(p)}\right\}$ is guaranteed by the 


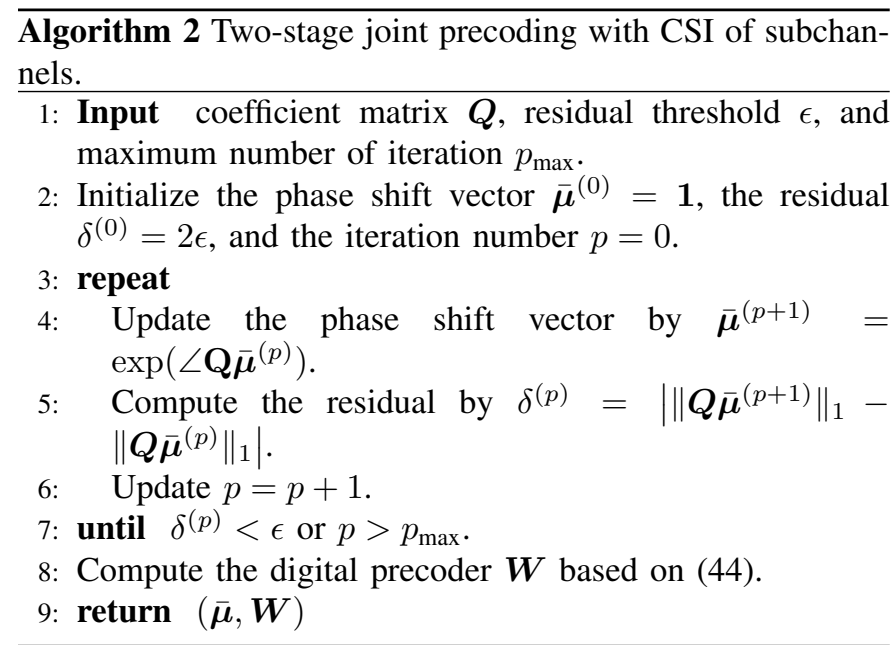

monotone convergence theorem [42]. By defining the residual as $\delta^{(p)}=\left\|\boldsymbol{Q} \overline{\boldsymbol{\mu}}^{(p+1)}\right\|_{1}-\left\|\boldsymbol{Q} \overline{\boldsymbol{\mu}}^{(p)}\right\|_{1}$, the stopping criterion given by $\delta^{(p)}<\epsilon$ is valid.

Let $\boldsymbol{v}$ be the limit of $\left\{\overline{\boldsymbol{\mu}}^{(p)}\right\}$, i.e., $\lim _{p \rightarrow \infty} \overline{\boldsymbol{\mu}}^{(p)}=\boldsymbol{\mu}^{\infty}$. Based on (43), we have $\boldsymbol{\mu}^{\infty}=e^{\mathrm{j} \angle\left(\boldsymbol{Q} \boldsymbol{\mu}^{\infty}\right)}$, which can be rewritten as

$$
\boldsymbol{Q} \boldsymbol{\mu}^{\infty}=\operatorname{diag}\left(\left|\boldsymbol{Q} \boldsymbol{\mu}^{\infty}\right|\right) e^{\mathrm{j} \angle\left(\boldsymbol{Q} \boldsymbol{\mu}^{\infty}\right)}=\operatorname{diag}\left(\left|\boldsymbol{Q} \boldsymbol{\mu}^{\infty}\right|\right) \boldsymbol{\mu}^{\infty} .
$$

Thus, the KKT condition (42) is satisfied by the fixed-point based method.

The computational complexity is dominated by computing the coefficient matrix $Q$ and iteratively solving the KKT condition (42). The former part has a complexity of $\mathcal{O}\left(M^{2} N_{\mathrm{t}} N_{\mathrm{s}}^{2}\right)$ on determining $\operatorname{tr}\left(\boldsymbol{P}_{n}^{\mathrm{H}} \boldsymbol{P}_{m}\right)$ for $\forall m \geq n$, while the latter part in the worst case yields a complexity of $\mathcal{O}\left(M^{2} p_{\max }\right)$. Therefore, the total complexity of the fixed-point based method is given by $\mathcal{O}\left(M^{2}\left(N_{\mathrm{t}} N_{\mathrm{s}}^{2}+p_{\max }\right)\right)$.

\section{Optimality of the Proposed Design in a Simple Scenario}

In this subsection, we show that the proposed two-stage design is capable of attaining the optimal precoding performance in a simple scenario, where the direct link between the transmitter and the receiver is blocked, and both the incident and the reflected channels are LOS-dominated. This implies that we have $\boldsymbol{H}_{0} \approx \mathbf{0}, K_{\mathrm{t}} \rightarrow \infty$, and $K_{\mathrm{r}} \rightarrow \infty$, with $K_{\mathrm{t}}$ and $K_{\mathrm{r}}$ being the Rician factors of $\boldsymbol{H}_{\mathrm{t}}$ and $\boldsymbol{H}_{\mathrm{r}}$, respectively. Moreover, it is also illustrated that, under the channel conditions considered, the optimal precoding scheme purely relies on the AOAs and AODs of $\boldsymbol{H}_{\mathrm{r}}$ and $\boldsymbol{H}_{\mathrm{t}}$, which has the potential of eliminating the channel estimation overhead with the aid of angular information.

It is noted that the design of $(\overline{\boldsymbol{\mu}}, \boldsymbol{W})$ given by Algorithm 2, in general, is only a near-optimal solution to (P2), which has a performance loss bounded by (36). However, we show that in this specific case, the proposed precoding design is actually capable of reaching the upper bound given by $\tilde{I}\left(\boldsymbol{P}^{\mathrm{opt}}\right)$, therefore achieving the optimal performance. Moreover, we reveal that under this scenario, the reflected link provides a reflection gain that is proportional to the square of the number of REs.
For convenience, we denote $\boldsymbol{H}_{\mathrm{r}}=\sqrt{\beta_{\mathrm{r}}} \boldsymbol{a} \boldsymbol{b}^{\mathrm{H}}$, and $\boldsymbol{H}_{\mathrm{t}}=$ $\sqrt{\beta_{\mathrm{t}}} \boldsymbol{c} \boldsymbol{d}^{\mathrm{H}}$, where $\beta_{\mathrm{r}}$ and $\beta_{\mathrm{t}}$ are the path loss coefficients, and $\boldsymbol{a}, \boldsymbol{b}, \boldsymbol{c}$, and $\boldsymbol{d}$ are the corresponding array response vectors given by (3), with the AOAs and AODs omitted for simplicity. The $m$ th subchannel is expressed as $\boldsymbol{H}_{m}=\sqrt{\beta_{\mathrm{r}} \beta_{\mathrm{t}}} t_{m} \boldsymbol{a d} \boldsymbol{d}^{\mathrm{H}}$, with $t_{m}=[\boldsymbol{b}]_{m}^{*}[\boldsymbol{c}]_{m}$, for $1 \leq m \leq M$, and $\boldsymbol{H}_{\mathrm{eq}}$ is rewritten as

$$
\boldsymbol{H}_{\mathrm{eq}}=\sqrt{\beta_{\mathrm{r}} \beta_{\mathrm{t}}} \boldsymbol{t}^{\mathrm{T}} \otimes \boldsymbol{a d}^{\mathrm{H}},
$$

where $\boldsymbol{t}=\left[\begin{array}{lll}t_{1} & \ldots, t_{M}\end{array}\right]^{\mathrm{T}}$ is an $M \times 1$ vector.

Before showing the optimality of the proposed precoding design in Proposition 6, we present a lemma as follows

Lemma 2: The optimal solution to the joint precoding problem $(\mathrm{P} 2)$ is given by

$$
\left(\boldsymbol{\mu}^{\mathrm{opt}}, \boldsymbol{w}^{\mathrm{opt}}\right)=\left(\boldsymbol{t}^{*}, \frac{1}{\sqrt{N_{\mathrm{t}}}} \boldsymbol{d}\right)
$$

and the optimal value of (P2) matches its upper bound provided by $\boldsymbol{p}^{\text {opt }}$, i.e.,

$$
I\left(\boldsymbol{\mu}^{\mathrm{opt}}, \boldsymbol{w}^{\mathrm{opt}}\right)=\tilde{I}\left(\boldsymbol{p}^{\mathrm{opt}}\right) .
$$

Proof: Firstly, we derive $\boldsymbol{p}^{\text {opt }}$ from the eigenvectors of $\boldsymbol{H}_{\mathrm{eq}}^{\mathrm{H}} \boldsymbol{H}_{\mathrm{eq}}$. Denoting the eigenvalue and the corresponding eigenvector of $\boldsymbol{H}_{\mathrm{eq}}^{\mathrm{H}} \boldsymbol{H}_{\mathrm{eq}}$ by $\gamma$ and $\boldsymbol{f}$, respectively, we have $\boldsymbol{H}_{\mathrm{eq}}^{\mathrm{H}} \boldsymbol{H}_{\mathrm{eq}} \boldsymbol{f}=\gamma \boldsymbol{f}$, which can be rearranged as

$$
\left(\boldsymbol{t}^{*} \boldsymbol{t}^{\mathrm{T}}\right) \otimes\left(\boldsymbol{d} \boldsymbol{d}^{\mathrm{H}}\right) \boldsymbol{f}=\frac{\gamma}{\beta_{\mathrm{r}} \beta_{\mathrm{t}} \boldsymbol{a}^{\mathrm{H}} \boldsymbol{a}} \boldsymbol{f} .
$$

For convenience, let $\boldsymbol{f}=\left[\begin{array}{lll}\tilde{\boldsymbol{f}}_{1}^{\mathrm{T}} & \ldots, \tilde{\boldsymbol{f}}_{M}^{\mathrm{T}}\end{array}\right]^{\mathrm{T}}$, where $\tilde{\boldsymbol{f}}_{m}, 1 \leq$ $m \leq M$, is an $N_{\mathrm{t}} \times 1$ vector. Due to the Kronecker product structure of $\left(\boldsymbol{t}^{*} \boldsymbol{t}^{\mathrm{T}}\right) \otimes\left(\boldsymbol{d} \boldsymbol{d}^{\mathrm{H}}\right)$, Equation (50) holds only if $\tilde{\boldsymbol{f}}_{m}$ is linearly dependent on $\boldsymbol{d}$, i.e., $\tilde{\boldsymbol{f}}_{m}=l_{m} \boldsymbol{d}$. Thus, (50) can be further transformed to

$$
\boldsymbol{t}^{*} \boldsymbol{t}^{\mathrm{T}} \boldsymbol{l}=\frac{\gamma}{\beta_{\mathrm{r}} \beta_{\mathrm{t}}\left(\boldsymbol{a}^{\mathrm{H}} \boldsymbol{a}\right)\left(\boldsymbol{d}^{\mathrm{H}} \boldsymbol{d}\right)} \boldsymbol{l},
$$

where $\boldsymbol{l}=\left[l_{1}, \ldots, l_{M}\right]^{\mathrm{T}}$. Without loss of generality, this equation is solved by $\boldsymbol{l}=\boldsymbol{t}^{*}$. Observe that Equation (51) has a rank-one solution space, and thus $\boldsymbol{P}^{\text {opt }}$ is reduced to an $M N_{\mathrm{t}} \times 1$ vector:

$$
\boldsymbol{p}^{\mathrm{opt}}=\frac{1}{\sqrt{N_{\mathrm{t}}}} \boldsymbol{f}=\boldsymbol{t}^{*} \otimes \frac{\boldsymbol{d}}{\sqrt{N_{\mathrm{t}}}},
$$

where $\frac{1}{\sqrt{N_{\mathrm{t}}}}$ is a normalization factor such that $\left\|\boldsymbol{p}^{\mathrm{opt}}\right\|_{2}^{2}=M$.

It is observed from (52) that $\boldsymbol{p}^{\text {opt }}$ is decomposed into the Kronecker product of the vectors $\boldsymbol{t}^{*}$ and $\frac{d}{\sqrt{N_{\mathrm{t}}}}$. Moreover, $\left|t_{m}\right|=\left|[\boldsymbol{b}]_{m}^{*}[\boldsymbol{c}]_{m}\right|=1, \frac{1}{N_{\mathrm{t}}}\|\boldsymbol{d}\|_{2}^{2}=1$, and hence the optimal solution to the joint precoding problem is obtained by $\left(\boldsymbol{\mu}^{\mathrm{opt}}, \boldsymbol{w}^{\mathrm{opt}}\right)=\left(\boldsymbol{t}^{*}, \frac{1}{\sqrt{N_{\mathrm{t}}}} \boldsymbol{d}\right)$. In this case, the upper bound of (P2) is matched, i.e., $I\left(\boldsymbol{\mu}^{\mathrm{opt}}, \boldsymbol{w}^{\mathrm{opt}}\right)=\tilde{I}\left(\boldsymbol{p}^{\mathrm{opt}}\right)$.

Proposition 6: Let $\left(\boldsymbol{\mu}^{\mathrm{AL} 2}, \boldsymbol{w}^{\mathrm{AL} 2}\right)$ be the output of $\mathrm{Al}-$ gorithm 2. Then the proposed precoding method given in Algorithm 2 is capable of finding the optimal solution to (P2), i.e.,

$$
\left(\boldsymbol{\mu}^{\mathrm{AL} 2}, \boldsymbol{w}^{\mathrm{AL} 2}\right)=\left(\boldsymbol{\mu}^{\mathrm{opt}}, \boldsymbol{w}^{\mathrm{opt}}\right) .
$$

Proof: The entries of the coefficient matrix $Q$ in (P5) are given by $q_{m n}=\frac{1}{N_{\mathrm{t}}} t_{n} t_{m}^{*} \boldsymbol{d}^{\mathrm{H}} \boldsymbol{d}=t_{m}^{*} t_{n}$. Then the KKT condition (42) can be rewritten as

$$
\boldsymbol{t}^{*} \boldsymbol{t}^{\mathrm{T}} \boldsymbol{\mu}=\operatorname{diag}(\nu) \boldsymbol{\mu}
$$


Since $\boldsymbol{u}=\boldsymbol{t}^{*}$ is the unique solution to (54), it follows from invoking Proposition 5 that $\boldsymbol{\mu}^{\mathrm{AL} 2}=\boldsymbol{t}^{*}$. Then we have $\boldsymbol{H}_{\mathrm{eq}}\left(\boldsymbol{\mu}^{\mathrm{AL} 2} \otimes \boldsymbol{I}_{N_{\mathrm{t}}}\right)=\sqrt{\beta_{\mathrm{r}} \beta_{\mathrm{t}}} \boldsymbol{t}^{\mathrm{T}} \boldsymbol{t}^{*} \boldsymbol{a} \boldsymbol{d}^{\mathrm{H}}$, based on which the digital precoder is given by $\boldsymbol{w}^{\mathrm{AL} 2}=\frac{1}{\sqrt{N_{\mathrm{t}}}} \boldsymbol{d}$. Thus, we have $\left(\boldsymbol{\mu}^{\mathrm{AL} 2}, \boldsymbol{w}^{\mathrm{AL} 2}\right)=\left(\boldsymbol{\mu}^{\mathrm{opt}}, \boldsymbol{w}^{\mathrm{opt}}\right)$.

Based on the results of Lemma 2 and Proposition 6, the maximized MI of the RIS-aided system is achieved by the optimal design $\left(\boldsymbol{\mu}^{\mathrm{opt}}, \boldsymbol{w}^{\mathrm{opt}}\right)$, which is formulated as

$$
\begin{aligned}
I\left(\boldsymbol{\mu}^{\mathrm{opt}}, \boldsymbol{w}^{\mathrm{opt}}\right) & =\log _{2}\left|\boldsymbol{I}_{N_{\mathrm{r}}}+\frac{\rho \beta_{\mathrm{r}} \beta_{\mathrm{t}} M^{2} N_{\mathrm{t}}}{\sigma^{2}} \boldsymbol{a}^{\mathrm{H}}\right| \\
& =\log _{2}\left(1+\frac{\rho \beta_{\mathrm{r}} \beta_{\mathrm{t}}}{\sigma^{2}} N_{\mathrm{r}} N_{\mathrm{t}} M^{2}\right) .
\end{aligned}
$$

It is observed from (55) that the reflected link of RIS-aided systems exploits an extra reflection gain $M^{2}$ in addition to the conventional antenna array gains of $N_{\mathrm{r}} N_{\mathrm{t}}$. The reflection gain is proportional to the square of the number of REs, because each of the RIS RE serves as a mirror that produces a reflection of the transmitted signal. Given the optimal design of $\left(\boldsymbol{\mu}^{\mathrm{opt}}, \boldsymbol{w}^{\mathrm{opt}}\right)$, the signals represented by all of these reflections are aligned and superposed at the receiver. Consequently, it appears as if there were $M$ replica of the transmitter acting in union by increasing both the number of the TAs and the transmission power by a factor of $M$.

Additionally, observe that the optimal design is determined only by the array response vectors, i.e., $\left(\boldsymbol{\mu}^{\mathrm{opt}}, \boldsymbol{w}^{\mathrm{opt}}\right)=$ $\left(\boldsymbol{t}^{*}, \frac{1}{\sqrt{N_{\mathrm{t}}}} \boldsymbol{d}\right)$, with $t_{m}=[\boldsymbol{b}]_{m}^{*}[\boldsymbol{c}]_{m}$, for $1 \leq m \leq M$. Thus, the joint precoding problem can be solved by only relying on the knowledge of the AOA and AOD between the transmitter and the RIS, as well as those between the RIS and the receiver, which considerably reduces the complexity.

It should be noted that the channel conditions characterized in (47) are not perfectly satisfied in general cases. On this occasion, the upper bound $\tilde{I}\left(\boldsymbol{p}^{\mathrm{opt}}\right)$ is not attainable with any joint precoding design due to the degree-of-freedom reduction imposed by the Kronecker product structure of the phase shift vector and the digital precoder. Hence, the reflection gain of $M^{2}$ may not be fully exploited. Moreover, even if the reflection gain is fully exploited, the actual signal power of the reflected link at the receiver may still be lower than that of the direct link. This is because the reflected link generally experiences a much more substantial path loss, i.e., $\beta_{\mathrm{r}} \beta_{\mathrm{t}} \ll \beta_{0}$ [43]. The actual performance of the RIS-aided MIMO system will be evaluated for various channel realizations in the next section.

\section{NumericAl Results}

In this section, the performance of the proposed subchannel estimation method and the joint precoding scheme is investigated. We consider a RIS-aided MIMO system, where the transmitter is equipped with an $\sqrt{N_{\mathrm{t}}} \times \sqrt{N_{\mathrm{t}}}$ antenna array located at $\left(x_{\mathrm{t}}, y_{\mathrm{t}}\right)$, and the RIS having $M$ REs is placed at $\left(x_{\mathrm{RIS}}, y_{\mathrm{RIS}}\right)$. The signals transmitted from both the direct and the reflected links are received by the receiver at $\left(x_{\mathrm{r}}, y_{\mathrm{r}}\right)$, using an $\sqrt{N_{\mathrm{r}}} \times \sqrt{N_{\mathrm{r}}}$ antenna array. The antenna spacing of $D=\lambda / 2$ is adopted for all the UPAs. The noise power spectral density is $-174 \mathrm{dBm} / \mathrm{Hz}$, and the bandwidth is $200 \mathrm{kHz}$, which yields $\sigma^{2}=-121 \mathrm{dBm}$. The path loss for all channels is modeled as $\beta=\beta_{\text {ref }}\left(d / d_{\text {ref }}\right)^{-\alpha}$, where $\beta_{\text {ref }}=-30 \mathrm{~dB}$ is the path loss at the reference distance of $d_{\text {ref }}=1 \mathrm{~m}$, and $\alpha$ is the path loss exponent. The path loss for the direct channel, the incident channel and the reflected channel is denoted by $\beta_{0}, \beta_{\mathrm{t}}$ and $\beta_{\mathrm{r}}$, respectively, with the corresponding exponents being $\alpha_{0}=3.5, \alpha_{\mathrm{t}}=2.2$ and $\alpha_{\mathrm{r}}=2.8$ [17]. The path loss is calculated for $\left(x_{\mathrm{t}}, y_{\mathrm{t}}\right)=(0,0)$, $\left(x_{\mathrm{r}}, y_{\mathrm{r}}\right)=(200 \mathrm{~m}, 0)$ and $\left(x_{\mathrm{RIS}}, y_{\mathrm{RIS}}\right)=(10 \mathrm{~m}, 10 \mathrm{~m})$. The Rician fading model in (2) is used to generate the involved channels. Let $K_{0}, K_{\mathrm{t}}$, and $K_{\mathrm{r}}$ be the Rician factors of the direct, incident and reflected channels, respectively. The rich scattering propagation condition is characterized by $K=0$, while $K \rightarrow \infty$ indicates that the channel is LOS-dominated. All the results are averaged over 1000 channel realizations.

\section{A. Subchannel Estimation}

The performance of Algorithm 1 is evaluated by calculating the MSE for each different number $L$ of pilot training rounds and for each value $\rho$ of the transmission power. By setting $\tau=N_{\mathrm{t}}, \boldsymbol{X}=\sqrt{\rho / N_{\mathrm{t}}} \boldsymbol{F}_{N_{\mathrm{t}}}, M=7 \times 9, N_{\mathrm{t}}=N_{\mathrm{r}}=4$, $K_{0}=K_{\mathrm{t}}=K_{\mathrm{r}}=10 \mathrm{~dB}$, we compare the MSE obtained using the proposed subchannel estimation method under various implementations of the codebook $\boldsymbol{A}$, to its counterpart achieved by different benchmark schemes as follows:

- The DFT codebook specified in Corollary 1, labeled as "DFT codebook".

- The Hadamard codebook of Corollary 2, labeled as "Proposed Hadamard codebook".

- The trivial design using $\boldsymbol{A}=\boldsymbol{\Sigma}_{0}$ according to (19), labeled as "Trivial codebook".

- The random design using $[\boldsymbol{A}]_{m, n}=e^{\mathrm{j} \angle\left(\xi_{m, n}\right)}$ for $\forall m, n$, where $\xi_{m, n} \sim \mathcal{C N}(0,1)$, labeled as "Random codebook". 14

- The CRLB is calculated based on $\boldsymbol{\Theta}_{\mathrm{CRLB}}$ in Lemma 1, labeled as "CRLB".

- The least squared Khatri-Rao factorization (LSKRF) scheme proposed in [36] that estimates the full CSI of $\boldsymbol{H}_{\mathrm{r}}$ and $\boldsymbol{H}_{\mathrm{t}}$, labeled as "LSKRF scheme [36]".

- The cascaded channel estimation scheme proposed in [32] that adopts a DFT based LS training, labeled as "Cascaded CSI acquisition [32]".

By setting $\rho=0 \mathrm{dBm}$, the MSE performance of various codebooks is compared in Fig. 3(a), for different values of $L$. The initial value of $L$ in Fig. 3(a) is set to $L=M+1=64$, for satisfying the constraint of (18) that ensures the feasibility of the subchannel estimation method. ${ }^{15}$ It is observed that all the codebooks considered achieve a low MSE that is below $-80 \mathrm{~dB}$, which validates the accuracy of the proposed multi-round training scheme used for subchannel estimation. Additionally, the DFT/Hadamard codebooks outperform the other codebooks, and attain the CRLB curve. This observation

\footnotetext{
${ }^{14}$ By adopting a random codebook, the RIS configures its phase adjustment randomly in each round of pilot training. This does not affect the feasibility of the subchannel estimation method, but will result in a different MSE performance.

${ }^{15}$ As discussed in [36], the minimum overhead of the tensor-based LSKRF scheme is $M N_{\mathrm{t}}$ to estimate $\boldsymbol{H}_{\mathrm{t}}$ and $\boldsymbol{H}_{\mathrm{r}}$, and thus it is also required $L \geq$ $M+1$ for estimating $\boldsymbol{H}_{\mathrm{t}}, \boldsymbol{H}_{\mathrm{r}}$ and $\boldsymbol{H}_{0}$. The corresponding MSE is defined as $\operatorname{MSE}\left(\hat{\boldsymbol{H}}_{\mathrm{t}}, \hat{\boldsymbol{H}}_{\mathrm{r}}, \hat{\boldsymbol{H}}_{0}\right)=\mathrm{E}\left\{\left\|\hat{\boldsymbol{H}}_{\mathrm{t}}-\boldsymbol{H}_{\mathrm{t}}\right\|^{2}+\left\|\hat{\boldsymbol{H}}_{\mathrm{r}}-\boldsymbol{H}_{\mathrm{r}}\right\|^{2}+\left\|\hat{\boldsymbol{H}}_{0}-\boldsymbol{H}_{0}\right\|^{2}\right\}$, where $\hat{\boldsymbol{H}}_{\mathrm{t}}, \hat{\boldsymbol{H}}_{\mathrm{r}}$, and $\hat{\boldsymbol{H}}_{0}$ are the estimates of $\boldsymbol{H}_{\mathrm{t}}, \boldsymbol{H}_{\mathrm{r}}$, and $\boldsymbol{H}_{0}$, respectively.
} 


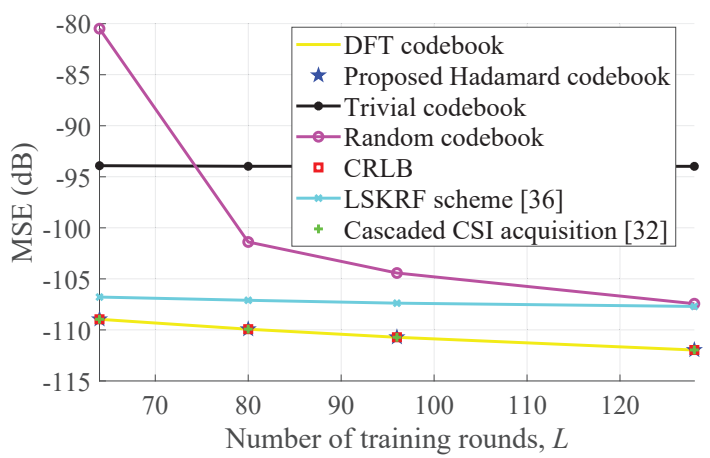

(a) MSE versus $L$, with $L \geq M+1$ and $\rho=0 \mathrm{dBm}$.

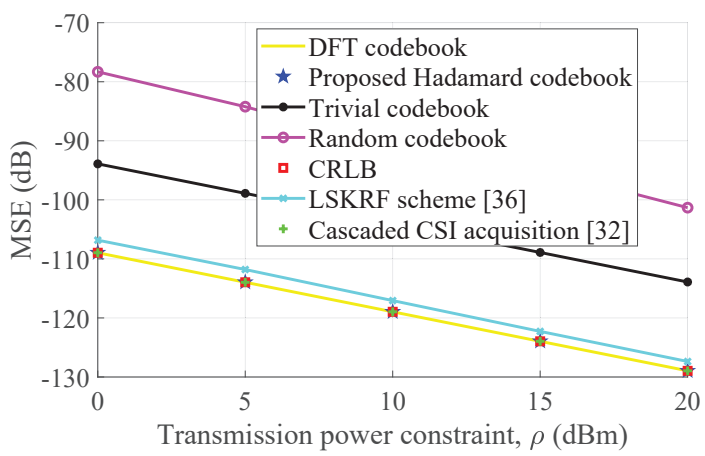

(b) MSE versus $\rho$, with $L=64$.

Fig. 3. MSE comparisons for different numbers of training rounds $L$, and various transmission power constraints $\rho$, where $N_{\mathrm{t}}=N_{\mathrm{r}}=4, M=7 \times 9$, and $K_{0}=K_{\mathrm{t}}=K_{\mathrm{r}}=10 \mathrm{~dB}$.

verifies the results given by Corollaries 1 and 2, where the DFT/Hadamard codebooks are proved to be optimal.

Given $L=64$, the MSE performance of different codebooks is illustrated in Fig. 3(b), versus the transmission power $\rho$. It is noted that both the DFT and the Hadamard codebooks improve the MSE performance of the subchannel estimator by about $15 \mathrm{~dB}$, compared to the trivial codebook. Furthermore, the random codebook shows the worst MSE performance, when using the minimum codebook length of $L=64$, which confirms the necessity of specifically designing the codebook for the RIS phase shifts configured during the multi-round training stage.

It is worth noting in Fig. 3(a) that the MSE of the LSKRF benchmark decreases slowly upon increasing $L$. This is because the MSE of the LSKRF scheme is contributed by not one, but two procedures: $(i)$ an LS estimation for the KhatriRao product of $\boldsymbol{H}_{\mathrm{t}}$ and $\boldsymbol{H}_{\mathrm{r}}$; (ii) a rank-1 approximation for obtaining each row $\backslash$ column of $\boldsymbol{H}_{\mathrm{t}} \backslash \boldsymbol{H}_{\mathrm{r}}$. Although the MSE imposed by $(i)$ can be further reduced given a larger $L$, the dominant part $(i i)$ cannot be eliminated by simply increasing $L$, and thus limits the overall performance. This also explains the observation from Fig. 3(b) that the proposed subchannel estimation method using DFT $\backslash$ Hadamard codebooks achieves better MSE performance than the LSKRF based benchmark, since the MSE of the proposed scheme is purely decided by the LS estimates of the subchannels. Additionally, the benchmark of cascaded CSI acquisition [32] shows a similar performance to the proposed scheme using DFT $\backslash$ Hadamard codebooks, because it also adopts a DFT based LS training.

\section{B. Joint Precoding Performance}

The performance of the proposed two-stage joint precoding method is evaluated for different numbers of RIS elements $M$, various transmission powers $\rho$, and diverse channel conditions specified by the Rician factors. The proposed scheme detailed in Algorithm 2 is compared to the following three benchmark precoding schemes:

- AO scheme [17]: the AO scheme derived in [17] is adopted, and the full CSI of the incident channel $\boldsymbol{H}_{\mathrm{t}}$ and the reflected channel $\boldsymbol{H}_{\mathrm{r}}$ is required.

- Random phase design: the phase shifts are randomly generated, and the digital precoder is determined by solving (P5) based on the random phase shifts.

- W/O RISs: the conventional MIMO system without RISs is considered, where the digital precoder is given by the solution to (P5) with $[\overline{\boldsymbol{\mu}}]_{(2: M+1)}=\mathbf{0}$.

Accounting for the overhead of channel estimation, the MI loss factor is given as $\left(1-\frac{\kappa_{s} N_{\mathrm{p}}}{\kappa_{c}}\right)$, where $\kappa_{c}$ is the length of the channel coherence interval, $\kappa_{s}$ is the duration of a single pilot symbol, and $N_{\mathrm{p}}$ is the overall number of pilot symbols required. We use the parameter values $\left(\kappa_{c}, \kappa_{s}\right)=$ $(0.025 \mathrm{~s}, 10 \mu \mathrm{s})$. For the proposed precoding method and the AO benchmark, $N_{\mathrm{p}}=(M+1) N_{\mathrm{t}}$ is adopted, while for the random phase design and the W/O RISs benchmark, $N_{\mathrm{p}}=N_{\mathrm{t}}$ is assumed. ${ }^{16}$ If without explicit explanation, the loss factor is applied to all the numerical results in this section, namely that $\left(1-\frac{\kappa_{s} N_{\mathrm{p}}}{\kappa_{c}}\right) I(\overline{\boldsymbol{\mu}}, \boldsymbol{W})$ is used as our performance metric.

1) MI versus the number of REs: The MI achieved by the proposed two-stage joint precoding scheme is compared to that of various benchmarks for different numbers of REs and diverse channel conditions, as shown in Fig. 4, where $N_{\mathrm{t}}=$ $N_{\mathrm{r}}=4$, the transmission power is $\rho=20 \mathrm{dBm}$, and the number of REs is set to $M=10 m \times 10$ for $m=0, \ldots, 10$.

Observe that the MI of all the schemes increases upon decreasing the Rician factors of the channels involved, thanks to the rich scattering propagation that is appropriate for increasing the benefits of MIMO systems [45]. By comparing the performance of the random phase design and the conventional MIMO system without RISs, it is seen from Fig. 4(c) that, under the LOS-dominant propagation conditions, even the randomly generated phase design is capable of gleaning some reflection gain (the MI increased by about 20\%). However, in the case of rich scattering channels with $K=0$, as demonstrated in Fig. 4(a), the RIS has almost no benefits if the phase shifts are randomly adjusted. This is because the effective channel of the reflected link becomes negligible compared to the direct channel, as a result of the law of large numbers

$$
\frac{1}{M} \sum_{m=1}^{M} \mu_{m}\left[\boldsymbol{H}_{\mathrm{r}}\right]_{(:, m)}\left[\boldsymbol{H}_{\mathrm{t}}\right]_{(m,:)} \approx \mathrm{E}\left\{\mu_{m}\left[\boldsymbol{H}_{\mathrm{r}}\right]_{(:, m)}\left[\boldsymbol{H}_{\mathrm{t}}\right]_{(m,:)}\right\} .
$$

\footnotetext{
${ }^{16} \mathrm{The}$ AO benchmark of [17] requires the full CSI of $\boldsymbol{H}_{0}, \boldsymbol{H}_{\mathrm{r}}$, and $\boldsymbol{H}_{\mathrm{t}}$, which can be acquired using the tensor based technique discussed in [36], with a minimum overhead of $(M+1) N_{\mathrm{t}}$ pilots. As for the benchmark of random phase design, the number of required pilots is only $N_{\mathrm{t}}$, because the CSI of $\boldsymbol{H}_{\mathrm{r}}, \boldsymbol{H}_{\mathrm{t}}$ is not needed to determine the RIS phase adjustments.
} 


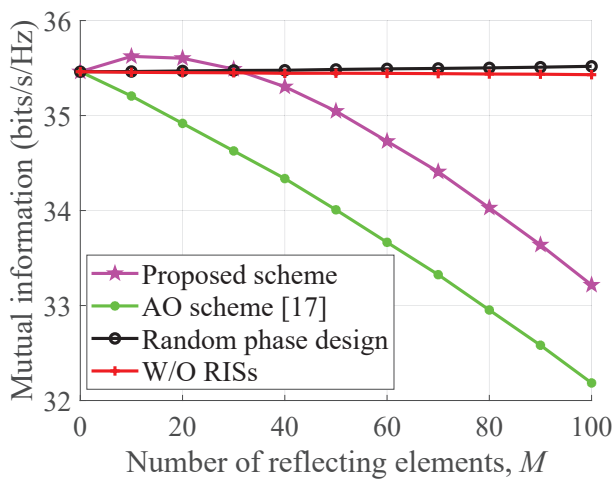

(a) $K_{0}, K_{\mathrm{t}}, K_{\mathrm{r}}=0$.

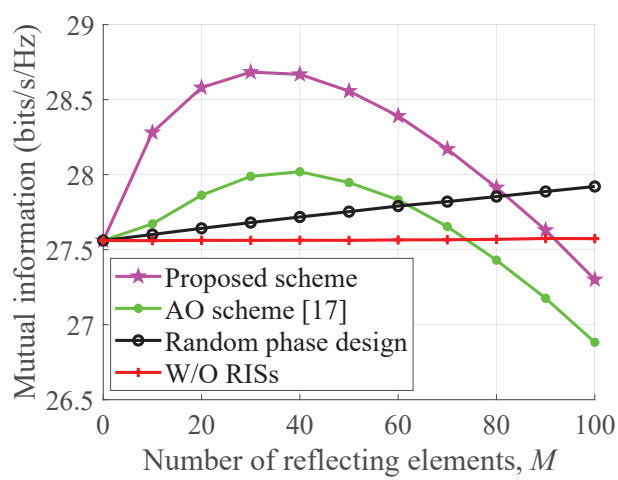

(b) $K_{0}, K_{\mathrm{t}}, K_{\mathrm{r}}=10 \mathrm{~dB}$.

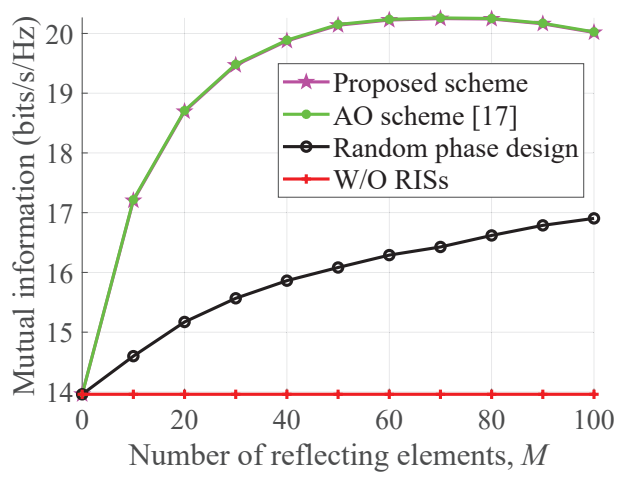

(c) $K_{0}, K_{\mathrm{t}}, K_{\mathrm{r}} \rightarrow \infty$.

Fig. 4. MI versus number of REs $M$, for various precoding designs, where $N_{\mathrm{t}}=N_{\mathrm{r}}=4$, and $\rho=20 \mathrm{dBm}$.

The expectation term of (56) is zero since $\mu_{m}, \boldsymbol{H}_{\mathrm{r}}$, and $\boldsymbol{H}_{\mathrm{t}}$ are independent, and the entries of $\boldsymbol{H}_{\mathrm{r}}$ and $\boldsymbol{H}_{\mathrm{t}}$ follow a zeromean complex symmetric Gaussian distribution.

It is also noted that the MI of both the proposed scheme and of the AO-based benchmark firstly increases and then decreases with $M$, under most channel conditions (except that the MI of the AO scheme keeps on decreasing in Fig. 4(a)). This is because when $M$ begins increasing, the resulted reflection gain provided by the RIS is exploited. However, the overhead of channel estimation becomes significant and thus limits the performance, when $M$ exceeds a certain value. As discussed in the context of (56), the performance gain achieved by the proposed scheme and by the $\mathrm{AO}$ scheme remains quite limited and decreases very quickly as $M$ increases under rich scattering conditions, which is due to the fact that the channel gain of the reflected link is negligible, given a large value of $M$, and yet the training overhead keeps on growing. Moreover, observe from Figs. 4(a) and 4(b) that the proposed two-stage precoding scheme outperforms the $\mathrm{AO}$ scheme in terms of increasing the MI.

2) MI versus the transmission power constraint: We set $N_{\mathrm{t}}=N_{\mathrm{r}}=4, M=3 \times 10$, and compare the MI of the proposed precoding scheme to those of the aforementioned benchmarks under different transmission power constraints and channel conditions, as illustrated in Fig. 5.

Observe in Fig. 5 that the MI obtained by all the schemes decreases upon increasing the Rician factors $K$. By contrast, increased reflection gain has been achieved in the presence of less scattering. Specifically, the proposed scheme outperforms the conventional MIMO system by about $0.5 \mathrm{~dB}$ in rich scattering environments, but its gain can be increased to more than $10 \mathrm{~dB}$ under the LOS-dominated channel conditions having $K \rightarrow \infty .{ }^{17}$ Additionally, the MI of all the compared schemes almost linearly increases with transmission power $\rho$ measured in $\mathrm{dBm}$. This observation verifies the conclusion of $I \propto \log \rho$ for point-to-point MIMO systems in the case of a high signal-to-noise ratio (SNR) at the receiver, due to the relatively modest path loss between the transmitter and the receiver in our simulation setting.

As illustrated in Figs. 5(a) and 5(b), the performance gain of the RIS-aided systems is very limited under rich scattering channels, especially after taking into account the realistic pilot overhead of channel estimation. Furthermore, the proposed two-stage scheme shows a slight advantage over the AO-based benchmark in maximizing the MI. These observations coincide with the results shown in Figs. 4(a) and 4(b), as seen from the zoomed-in windows of Fig. 5.

3) MI evaluation with subchannel grouping: By grouping the subchannels and using group-specific phase shifts, the complexity of both subchannel estimation and of the joint precoding is substantially reduced. For convenience, we set $N_{\mathrm{t}}=N_{\mathrm{r}}=4$, and $M=16 \times 16$. The index sets for the subchannel groups are determined by $\mathcal{S}_{0}=0$ and $\mathcal{S}_{g}=\left\{(g-1) M / 2^{R}+1, \ldots, g M / 2^{R}\right\}$, for $1 \leq g \leq N_{\mathrm{g}}-1$, where $R$ is a positive integer, and $R \leq \log _{2} M$. Explicitly, the direct channel $\boldsymbol{H}_{0}$ is assigned to Group 0, and the other $M$ subchannels are uniformly divided into $2^{R}$ groups. The total number of the groups is then given by $N_{\mathrm{g}}=2^{R}+1$.

We set $R \in\left\{0,1,2, \ldots, \log _{2} M\right\}$, and compare the MI obtained by the proposed two-stage precoding scheme under various Rician factors in Fig. 6. It is observed that the MI of the proposed scheme firstly increases with $R$ within a certain range, and this indicates that the group-specific phase shift design imposes considerable performance loss due to the phase ambiguity experienced. Quantitatively, for LOSdominated channels associated with $K \rightarrow \infty$, the grouping pattern of $2^{R}=1$, where all REs use an identical phase shift for the incident signal, results in about $30 \%$ performance degradation compared to the optimal grouping pattern of

\footnotetext{
${ }^{17}$ It is observed in Fig. 5(c) that the performance gain shown by the RISaided systems over the conventional MIMO systems increases upon increasing the power constraint. This is due to the fact that with $K \rightarrow \infty$, the direct channel spanning from the transmitter to the receiver becomes rank deficient [46]. Hence, the RIS-aided systems leveraging both the direct and reflected links achieves a higher multiplexing gain than the conventional systems using only the direct link.
} 


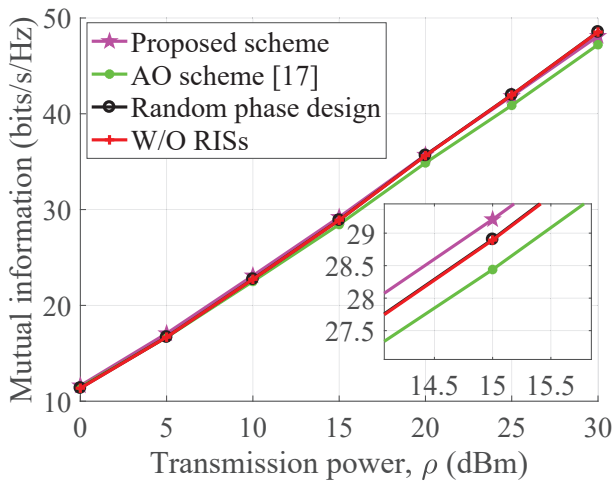

(a) $K_{0}, K_{\mathrm{t}}, K_{\mathrm{r}}=0$.

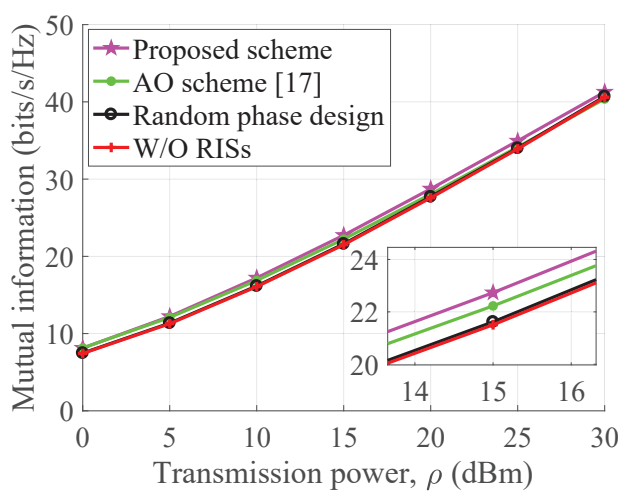

(b) $K_{0}, K_{\mathrm{t}}, K_{\mathrm{r}}=10 \mathrm{~dB}$

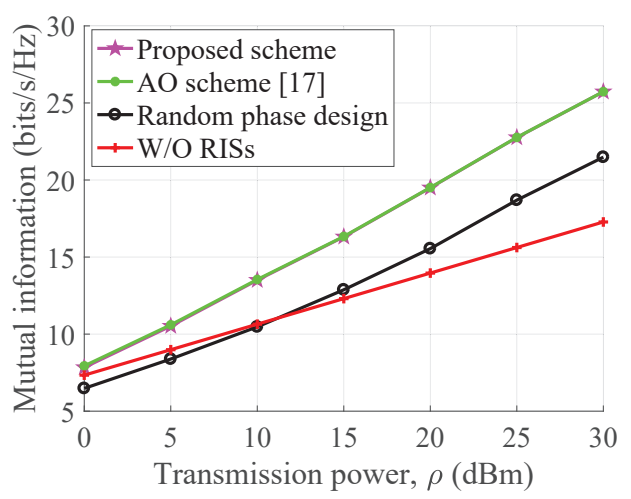

(c) $K_{0}, K_{\mathrm{t}}, K_{\mathrm{r}} \rightarrow \infty$.

Fig. 5. MI versus transmission power constraint $\rho$, for various precoding designs, where $N_{\mathrm{t}}=N_{\mathrm{r}}=4$, and $M=3 \times 10$.

$2^{R}=64$. This performance loss is a direct result of wave interference. When dispensing with grouping, the incident waves transmitted in every subchannel can be all phase-aligned by controlling the dedicated REs, which leads to constructive interference. As for the scenario of subchannel grouping, the incident waves corresponding to the same group have the same common phase shift, and hence they experience destructive interference.

It is also noted in Fig. 6 that when $R$ exceeds a certain value, the MI achieved by the proposed scheme decreases upon increasing $R$. This is due to the fact that the overhead of channel training, given by $\left(2^{R}+1\right) N_{\mathrm{t}}$, also increases with $R$. Therefore, the number of RE groups should be appropriately chosen in practical systems for striking an appealing reflection gain versus training overhead trade-off.

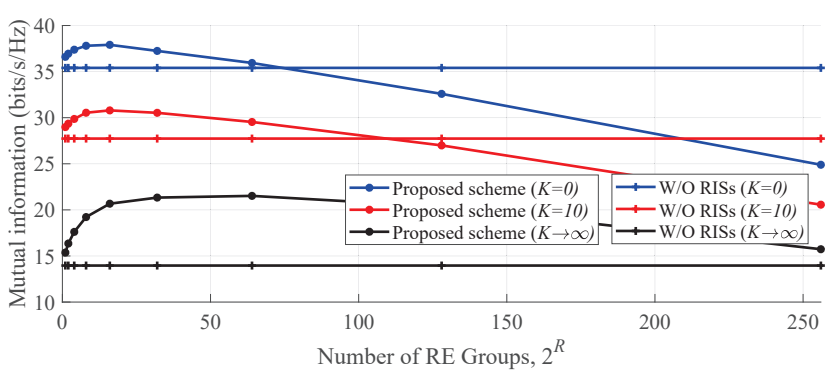

Fig. 6. MI versus number of RE groups $2^{R}$, for various subchannel grouping patterns, where Algorithm 2 is adopted for precoding design, $\rho=20 \mathrm{dBm}$, $N_{\mathrm{t}}=N_{\mathrm{r}}=4$, and $M=16 \times 16$.

4) Effects of channel estimation errors: The MI achieved by the proposed two-stage precoding method is shown in Fig. 7, relying on the estimated subchannel CSI (obtained from Algorithm 1) instead of the perfect CSI, under different transmission power constraints $\rho .^{18}$ The performance loss is calculated by $\left(I_{0}-\hat{I}_{0}\right) / I_{0}$ with $I_{0}$ and $\hat{I}_{0}$ denoting the MI obtained using perfect CSI and estimated CSI, respectively. It is seen that the performance loss decreases with the transmission power $\rho$, and it declines more rapidly given a larger $L$, i.e., more training rounds. This is because the estimated CSI becomes more accurate given more transmission power and pilot symbols. More specifically, the performance loss finally decreases to zero, as shown in Figs. 7(a) and 7(b). By contrast, the loss still remains in Fig. 7(c), where all the involved channels are LOS-dominant. This is due to the fact that even negligible noise changes the rank-deficient nature of the LOS-dominant channels, and thus affects the design of the precoding matrix $\boldsymbol{W}$ obtained with the aid of SVD in (44). In this case, as discussed in [13], robust precoding designs can be considered by investigating the expectation over the distribution of the channel estimation error.

\section{CONCLUSIONS}

A channel decomposition model has been proposed for CSI acquisition and joint precoding problems in RIS-aided MIMO systems. By decomposing the effective channel of the reflected link into multiple subchannels, we have developed a multiround pilot training mechanism to obtain the CSI required for precoding. The joint precoding problem has been reformulated with respect to the subchannels, based on which a two-stage precoding scheme has been developed to successively design the reflecting phase shifts and the baseband digital precoder, only relying on the CSI knowledge of the subchannels. Our numerical results have demonstrated that the proposed channel decomposition based model provides an attractive solution for RIS-aided MIMO communications. For future works, joint transmit precoding and RIS phase adjustment under frequencyselective fading channels, as well as low-overhead methods for subchannel estimation are worth further investigation.

\section{APPENDIX A}

\section{PROOF OF LEMMA 1}

Proof: For convenience, let $\boldsymbol{C}=\boldsymbol{A}^{\mathrm{H}} \otimes \boldsymbol{I}_{N_{\mathrm{r}}}, \boldsymbol{D}=$ $\boldsymbol{X}^{\mathrm{H}}\left(\boldsymbol{X} \boldsymbol{X}^{\mathrm{H}}\right)^{-1}, \quad \boldsymbol{E}=\left[\begin{array}{lll}\hat{\boldsymbol{H}}_{\mathrm{e}, 1}^{\mathrm{T}}, & \ldots, & \hat{\boldsymbol{H}}_{\mathrm{e}, L}^{\mathrm{T}}\end{array}\right]^{\mathrm{T}}$, and $\boldsymbol{J}=$

\footnotetext{
${ }^{18}$ In order to present the effects of estimation errors under different numbers $L$ of the training rounds, the pilot overhead is not included in Fig. 7 .
} 


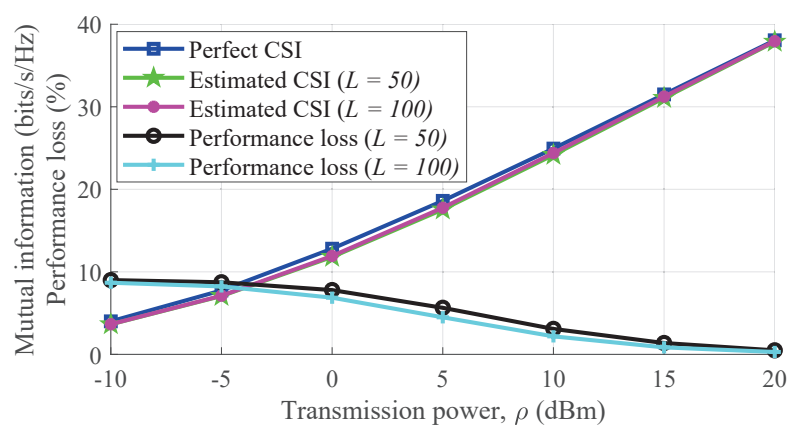

(a) $K_{0}, K_{\mathrm{t}}, K_{\mathrm{r}}=0$.

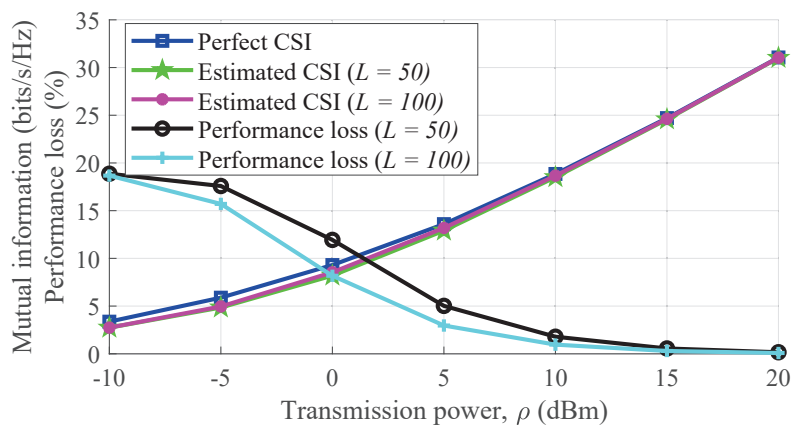

(b) $K_{0}, K_{\mathrm{t}}, K_{\mathrm{r}}=10 \mathrm{~dB}$

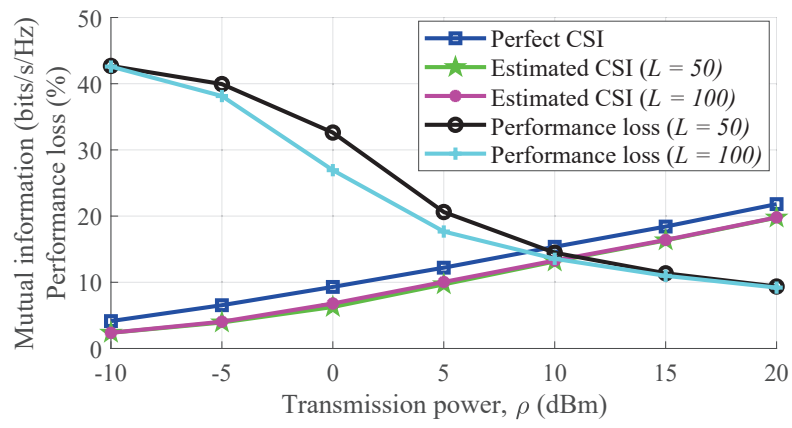

(c) $K_{0}, K_{\mathrm{t}}, K_{\mathrm{r}} \rightarrow \infty$.

Fig. 7. MI versus transmission power constraint $\rho$, relying on estimated CSI of the subchannels, where the performance loss is shown by percentage, $N_{\mathrm{t}}=N_{\mathrm{r}}=4$, and $M=7 \times 7$.

$\left[\boldsymbol{Z}_{1}^{\mathrm{T}}, \ldots, \boldsymbol{Z}_{L}^{\mathrm{T}}\right]^{\mathrm{T}}$. Then (9) can be rewritten as

$$
\operatorname{vec}(\boldsymbol{E})=\left(\boldsymbol{I}_{N_{\mathrm{t}}} \otimes \boldsymbol{C}\right) \operatorname{vec}\left(\boldsymbol{H}_{s}\right)+\left(\boldsymbol{D}^{\mathrm{T}} \otimes \boldsymbol{I}_{L N_{\mathrm{r}}}\right) \operatorname{vec}(\boldsymbol{J}),
$$

where $\boldsymbol{J} \sim \mathcal{C N}\left(\mathbf{0}, \sigma^{2} \boldsymbol{I}_{\tau L N_{\mathrm{r}}}\right)$. Based on the Gauss-Markov theorem [47], the CRLB covariance matrix derived from (57) is given by

$\boldsymbol{\Theta}_{\mathrm{CRLB}}=\sigma^{2}\left[\left(\boldsymbol{I}_{N_{\mathrm{t}}} \otimes \boldsymbol{C}\right)^{\mathrm{H}}\left(\boldsymbol{D}^{\mathrm{T}} \boldsymbol{D}^{*} \otimes \boldsymbol{I}_{L N_{\mathrm{r}}}\right)^{-1}\left(\boldsymbol{I}_{N_{\mathrm{t}}} \otimes \boldsymbol{C}\right)\right]_{(58)}^{-1}$.

By exploiting the properties of Kronecker product operations, (58) can be further rearranged as

$$
\begin{aligned}
\boldsymbol{\Theta}_{\mathrm{CRLB}} & =\sigma^{2}\left(\boldsymbol{D}^{\mathrm{H}} \boldsymbol{D}\right)^{*} \otimes\left(\boldsymbol{C}^{\mathrm{H}} \boldsymbol{C}\right)^{-1} \\
& =\sigma^{2}\left(\boldsymbol{X}^{*} \boldsymbol{X}^{\mathrm{T}}\right)^{-1} \otimes\left(\boldsymbol{A} \boldsymbol{A}^{\mathrm{H}}\right)^{-1} \otimes \boldsymbol{I}_{N_{\mathrm{r}}},
\end{aligned}
$$

which completes the proof.

\section{APPENDIX B}

\section{PROOF OF PROPOSITION 4}

Proof: For convenience, denote $\boldsymbol{E}=\rho / \sigma^{2} \boldsymbol{H}_{\mathrm{eq}}(\overline{\boldsymbol{\mu}} \otimes$ $\boldsymbol{W})(\overline{\boldsymbol{\mu}} \otimes \boldsymbol{W})^{\mathrm{H}} \boldsymbol{H}_{\mathrm{eq}}^{\mathrm{H}}, \boldsymbol{C}=\overline{\boldsymbol{\mu}} \otimes \boldsymbol{W}, \boldsymbol{D}=\boldsymbol{P}^{\mathrm{opt}}, \boldsymbol{B}=$ $\rho / \sigma^{2} \boldsymbol{H}_{\mathrm{eq}} \boldsymbol{P}^{\mathrm{opt}}\left(\boldsymbol{H}_{\mathrm{eq}} \boldsymbol{P}^{\mathrm{opt}}\right)^{\mathrm{H}}$, and $R=N_{\mathrm{s}}$. Then for any feasible $(\overline{\boldsymbol{\mu}}, \boldsymbol{W})$, the difference between the objective of (P2) and its upper bound $\tilde{I}\left(\boldsymbol{P}^{\mathrm{opt}}\right)$ is given by

$$
\begin{aligned}
& \left|I(\overline{\boldsymbol{\mu}}, \boldsymbol{W})-\tilde{I}\left(\boldsymbol{P}^{\mathrm{opt}}\right)\right| \\
& =\left|\sum_{r=1}^{R} \log \left(1+\lambda_{r}(\boldsymbol{E})\right)-\sum_{r=1}^{R} \log \left(1+\lambda_{r}(\boldsymbol{B})\right)\right| \\
& \leq \sum_{r=1}^{R}\left|\log \left(1+\lambda_{r}(\boldsymbol{E})\right)-\log \left(1+\lambda_{r}(\boldsymbol{B})\right)\right| \\
& \stackrel{(a)}{\leq} \sum_{r=1}^{R}\left|\lambda_{r}(\boldsymbol{E})-\lambda_{r}(\boldsymbol{B})\right| \stackrel{(b)}{\leq} \sqrt{R \sum_{r=1}^{R}\left|\lambda_{r}(\boldsymbol{E})-\lambda_{r}(\boldsymbol{B})\right|^{2}} \\
& \stackrel{(c)}{\leq} \sqrt{R}\|\boldsymbol{E}-\boldsymbol{B}\|_{F} \stackrel{(d)}{\leq} \frac{\rho \sqrt{R}}{\sigma^{2}}\left\|\boldsymbol{H}_{\mathrm{eq}}\right\|_{F}^{2}\left\|\boldsymbol{C} \boldsymbol{C}^{\mathrm{H}}-\boldsymbol{D} \boldsymbol{D}^{\mathrm{H}}\right\|_{F},
\end{aligned}
$$

where $(a)$ is a result of the property of logarithmic function, (b) follows from invoking the Cauchy-Schwarz inequality, and (c) is obtained by applying Theorem III.4.4 in [48]. Note that the result given by $(d)$ in (60) can be further bounded by

$$
\begin{aligned}
\| \boldsymbol{C} \boldsymbol{C}^{\mathrm{H}} & -\boldsymbol{D} \boldsymbol{D}^{\mathrm{H}} \|_{F} \\
& =\left\|(\boldsymbol{C}+\boldsymbol{D})(\boldsymbol{C}-\boldsymbol{D})^{\mathrm{H}}+\boldsymbol{C} \boldsymbol{D}^{\mathrm{H}}-\boldsymbol{D} \boldsymbol{C}^{\mathrm{H}}\right\|_{F} \\
& \leq\left\|(\boldsymbol{C}+\boldsymbol{D})(\boldsymbol{C}-\boldsymbol{D})^{\mathrm{H}}\right\|_{F}+\left\|\boldsymbol{C} \boldsymbol{D}^{\mathrm{H}}-\boldsymbol{D} \boldsymbol{C}^{\mathrm{H}}\right\|_{F},
\end{aligned}
$$

where the first term is bounded by $\left\|(\boldsymbol{C}+\boldsymbol{D})(\boldsymbol{C}-\boldsymbol{D})^{\mathrm{H}}\right\|_{F} \leq$ $2(M+1)\left\|\boldsymbol{C}-\boldsymbol{D}^{\mathrm{H}}\right\|_{F}$ due to $\|\boldsymbol{C}\|_{F}=\|\boldsymbol{D}\|_{F}=(M+1)$, while the second term is bounded by

$$
\begin{aligned}
\| \boldsymbol{C} \boldsymbol{D}^{\mathrm{H}} & -\boldsymbol{D C}^{\mathrm{H}} \|_{F} \\
& =\left\|\boldsymbol{C}(\boldsymbol{D}-\boldsymbol{C})^{\mathrm{H}}-(\boldsymbol{D}-\boldsymbol{C}) \boldsymbol{C}^{\mathrm{H}}\right\|_{F} \\
& \leq 2\left\|\boldsymbol{C}(\boldsymbol{D}-\boldsymbol{C})^{\mathrm{H}}\right\|_{F} \leq 2(M+1)\|\boldsymbol{C}-\boldsymbol{D}\|_{F} .
\end{aligned}
$$

Based on (60)-(62), letting $C=\frac{4 \rho}{\sigma^{2}} \sqrt{R}(M+1)\left\|\boldsymbol{H}_{\text {eq }}\right\|_{F}^{2}$, we have

$$
\left|I(\overline{\boldsymbol{\mu}}, \boldsymbol{W})-\tilde{I}\left(\boldsymbol{P}^{\mathrm{opt}}\right)\right| \leq C\left\|\overline{\boldsymbol{\mu}} \otimes \boldsymbol{W}-\boldsymbol{P}^{\mathrm{opt}}\right\|_{F} .
$$

\section{REFERENCES}

[1] S. Buzzi, C.-L. I, T. E. Klein, H. V. Poor, C. Yang, and A. Zappone, "A survey of energy-efficient techniques for $5 \mathrm{G}$ networks and challenges ahead", IEEE J. Sel. Areas Commun., vol. 34, no. 4, pp. 697-709, Apr. 2016.

[2] E. Basar, M. Di Renzo, J. De Rosny, M. Debbah, M.-S. Alouini, and R. Zhang, "Wireless communications through reconfigurable intelligent surfaces", IEEE Access, vol. 7, pp. 116753-116773, 2019.

[3] Q. Wu and R. Zhang, "Towards smart and reconfigurable environment: Intelligent reflecting surface aided wireless networks," IEEE Commun. Mag., vol. 58, no. 1, pp. 106-112, Jan. 2020.

[4] M. Di Renzo, M. Debbah, D.-T. Phan-Huy, A. Zappone, M.-S. Alouini, C.Yuen, V. Sciancalepore, G. C. Alexandropoulos, J. Hoydis, H. Gacanin, J. de Rosny, A. Bounceur, G. Lerosey, and M. Fink, "Smart radio environments empowered by reconfigurable AI meta-surfaces: An idea whose time has come," EURASIP J. Wireless Commun. Netw., vol. 2019, no. 1, p. 129, May 2019.

[5] C. Liaskos, S. Nie, A. Tsioliaridou, A. Pitsillides, S. Ioannidis, and I. Akyildiz, "A new wireless communication paradigm through softwarecontrolled metasurfaces," IEEE Commun. Mag., vol. 56, no. 9, pp. 162169, Sep. 2018.

[6] S. V. Hum and J. Perruisseau-Carrier, "Reconfigurable reflectarrays and array lenses for dynamic antenna beam control: A review," IEEE Trans. Antennas Propag., vol. 62, no. 1, pp. 183-198, Jan. 2014. 
[7] Z. Zhou, N. Ge, Z. Wang, and S. Chen, "Hardware-efficient hybrid precoding for millimeter wave systems with multi-feed reflectarrays," IEEE Access, vol. 6, pp. 6795-6806, Jan. 2018.

[8] V. Jamali, A. M. Tulino, G. Fischer, R. Muller, and R. Schober, "Scalable and energy-efficient millimeter massive MIMO architectures: Reflectarray and transmit-array antennas," in Proc. IEEE ICC, May 2019.

[9] C. Huang, A. Zappone, G. C. Alexandropoulos, M. Debbah, and C. Yuen, "Reconfigurable intelligent surfaces for energy efficiency in wireless communication," IEEE Trans. Wireless Commun., vol. 18, no. 8, pp. 41574170, Aug. 2019.

[10] W. Tang, et al., "Wireless communications with reconfigurable intelligent surface: Path loss modeling and experimental measurement," IEEE Trans. Wireless Commun., early access, doi: 10.1109/TWC.2020.3024887.

[11] M.-A. Badiu and J. P. Coon, "Communication through a large reflecting surface with phase errors," IEEE Wireless Commun. Lett., vol. 9, no. 2, pp. 184-188, Feb. 2020.

[12] Q. Wu and R. Zhang, "Intelligent reflecting surface enhanced wireless network via joint active and passive beamforming," IEEE Trans. Wireless Commun., vol. 18, no. 11, pp. 5394-5409, Nov. 2019.

[13] H. Guo, Y.-C. Liang, J. Chen, and E. G. Larsson, "Weighted sumrate maximization for reconfigurable intelligent surface aided wireless networks," IEEE Trans. Wireless Commun., vol. 19, no. 5, pp. 3064 3076, May 2020.

[14] B. Di, H. Zhang, L. Li, L. Song, Y. Li, and Z. Han, "Practical hybrid beamforming with finite-resolution phase shifters for reconfigurable intelligent surface based multi-user communications," IEEE Trans. Veh. Technol., vol. 64, no. 4, pp. 4565-4570, Apr. 2020.

[15] G. Zhou, C. Pan, H. Ren, K. Wang, and A. Nallanathan "Intelligent reflecting surface aided multigroup multicast MISO communication systems," IEEE Trans. Signal Process., vol. 68, pp. 3236-3251, 2020.

[16] N. S. Perović, M. Di Renzo, and M. F. Flanagan, "Channel capacity optimization using reconfigurable intelligent surfaces in indoor mmwave environments," in Proc. IEEE ICC, Jun. 2020.

[17] S. Zhang and R. Zhang, "Capacity characterization for intelligent reflecting surface aided MIMO communication," IEEE J. Sel. Areas Commun., vol. 38, no. 8, pp. 1823-1838, Aug. 2020.

[18] J. Ye, S. Guo, and M.-S. Alouini, "Joint reflecting and precoding designs for SER minimization in reconfigurable intelligent surfaces assisted MIMO systems," IEEE Trans. Wireless Commun., vol. 19, no. 8, pp. 55615574, Aug. 2020.

[19] C. Pan, H. Ren, K. Wang, W. Xu, M. Elkashlan, A. Nallanathan, and L. Hanzo, "Multicell MIMO communications relying on intelligent reflecting surfaces," IEEE Trans. Wireless Commun., vol. 19, no. 8, pp. 5218-5233, Aug. 2020.

[20] M. Di Renzo, A. Zappone, M. Debbah, M.-S. Alouini, C. Yuen, J De Rosny, and S. Tretyakov, "Smart radio environments empowered by reconfigurable intelligent surfaces: How it works, state of research, and road ahead," IEEE J. Sel. Areas Commun., early access, doi: 10.1109/JSAC.2020.3007211.

[21] C. Huang, S. Hu, G. C. Alexandropoulos, A. Zappone, C. Yuen, R. Zhang, M. Di Renzo, and M. Debbah, "Holographic MIMO surfaces for 6G wireless networks: Opportunities, challenges, and trends," IEEE Wireless Commun., early access, doi: 10.1109/MWC.001.1900534.

[22] A. Taha, M. Alrabeiah, and A. Alkhateeb, "Enabling large intelligent surfaces with compressive sensing and deep learning," [Online] Available: https://arxiv.org/abs/1904.10136.

[23] G. C. Alexandropoulos and E. Vlachos, "A hardware architecture for reconfigurable intelligent surfaces with minimal active elements for explicit channel estimation," in IEEE Int. Conf. Acoust., Speech and Signal Process. (ICASSP), pp. 9175-9179, May 2020.

[24] D. Mishra and H. Johansson, "Channel estimation and low-complexity beamforming design for passive intelligent surface assisted MISO wireless energy transfer," in IEEE Int. Conf. Acoust., Speech and Signal Process. (ICASSP), Brighton,UK, pp. 4659-4663, May 2019.

[25] B. Zheng and R. Zhang, "Intelligent reflecting surface-enhanced OFDM: Channel estimation and reflection optimization," IEEE Wireless Commun. Lett., vol. 9, no. 4, pp. 518-522, Apr. 2020.

[26] Y. Yang, B. Zheng, S. Zhang, R. Zhang, "Intelligent reflecting surface meets OFDM: Protocol design and rate maximization," IEEE Trans. Commun., vol. 68, no. 7, pp. 4522-4535, Jul. 2020.

[27] Q.-U.-A. Nadeem, H. Alwazani, A. Kammoun, A. Chaaban, M. Debbah, and M.-S. Alouini, "Intelligent reflecting surface-assisted multi-user MISO communication: Channel estimation and beamforming design," IEEE Open Journal of the Communications Society, vol. 1, pp. 661-680, 2020.
[28] P. Wang, J. Fang, H. Duan, and H. Li, "Compressed channel estimation for intelligent reflecting surface-assisted millimeter wave systems," IEEE Signal Process. Lett., vol. 27, pp. 905-909, 2020.

[29] Z.-Q. He and X. Yuan, "Cascaded channel estimation for large intelligent metasurface assisted massive MIMO," IEEE Wireless Commun. Lett., vol. 9, no. 2, pp. 210-214, Feb. 2020.

[30] H. Liu, X. Yuan, and Y.-J. A. Zhang, "Matrix-calibration-based cascaded channel estimation for reconfigurable intelligent surface assisted multiuser MIMO," IEEE J. Sel. Areas Commun., early access, doi: 10.1109/JSAC.2020.3007057.

[31] J. Chen, Y.-C. Liang, H. V. Cheng, and W. Yu, "Channel estimation for reconfigurable intelligent surface aided multi-user MIMO systems," [Online]. Available: https://arxiv.org/abs/1912.03619.

[32] T. L. Jensen and E. De Carvalho, "An optimal channel estimation scheme for intelligent reflecting surfaces based on a minimum variance unbiased estimator," in Proc. IEEE Int. Conf. Acoust., Speech Signal Process. (ICASSP), pp. 5000-5004, May 2020.

[33] Q. Wu and R. Zhang, "Beamforming optimization for wireless network aided by intelligent reflecting surface with discrete phase shifts," IEEE Trans. Commun., vol. 68, no. 3, pp. 1838-1851, Mar. 2020.

[34] Ö. Özdogan, E. Björnson, and J. Zhang, "Performance of cell-free massive MIMO with Rician fading and phase shifts," IEEE Trans. Wireless Commun., vol. 18, no. 11, pp. 5299-5315, Nov. 2019.

[35] E. Björnson, and L. Sanguinetti, "Rayleigh fading modeling and channel hardening for reconfigurable intelligent surfaces," [Online]. Available: https://arxiv.org/abs/2009.04723.

[36] G. T. de Araújo, and A. L. F. de Almeida, "PARAFAC-based channel estimation for intelligent reflective surface assisted MIMO system," in Proc. IEEE Sensor Array Multichannel Signal Process. Workshop (SAM), Jun. 2020.

[37] J.-M. Kang, "Intelligent reflecting surface: Joint optimal training sequence and refection pattern," IEEE Commun. Lett., vol. 24, no. 8, pp. 1784-1788, Aug. 2020.

[38] S. Guo, H. Zhang, P. Zhang, P. Zhao, L. Wang, and M.-S. Alouini, "Generalized beamspace modulation using multiplexing: A breakthrough in mmWave MIMO," IEEE J. Sel. Areas Commun., vol. 37, no. 9, pp. 2014-2028, Sep. 2019.

[39] R. W. Heath, N. Gonzáez-Prelcic, S. Rangan, W. Roh, and A. M. Sayeed, "An overview of signal processing techniques for millimeter wave MIMO systems," IEEE J. Sel. Topics in Signal Process., vol. 10, no. 3, pp. 436453, Apr. 2016.

[40] D. Zhang, Y. Wang, X. Li, and W. Xiang, "Hybridly connected structure for hybrid beamforming in mmWave massive MIMO systems," IEEE Trans. Commun., vol. 66, no. 2, pp. 662-674, Feb. 2018.

[41] O. E. Ayach, S. Rajagopal, S. Abu-Surra, Z. Pi, and R. W. Heath, "Spatially sparse precoding in millimeter wave MIMO systems," IEEE Trans. Wireless Commun., vol. 13, no. 3, pp. 1499-1513, Mar. 2014.

[42] G. B. Folland, Real Analysis: Modern Techniques and Their Applications, John Wiley \& Sons, 2013.

[43] E. Björnson, Ö. Özdogan, and E. G. Larsson, "Intelligent reflecting surface vs. decode-and-forward: How large surfaces are needed to beat relaying?" IEEE Wireless Commun. Lett., vol. 9, no. 2, pp. 244-248, Feb. 2020.

[44] X. Zhang, Matrix Analysis and Applications, Cambridge University Press, 2017.

[45] E. Björnson, E. G. Larsson, and T. L. Marzetta, "Massive MIMO: Ten myths and one critical question," IEEE Commun. Mag., vol. 54, no. 2, pp. 114-123, Feb. 2016.

[46] H. Q. Ngo, E. G. Larsson, and T. L. Marzetta, "Energy and spectral efficiency of very large multiuser MIMO systems," IEEE Trans. Commun., vol. 61, no. 4, pp. 1436-1449, Apr. 2013.

[47] S. Kay, Fundamentals of Statistical Signal Processing: Estimation Theory, Englewood Cliffs, NJ: Prentice-Hall, 1993.

[48] R. Bhatia, (2013). Matrix Analysis, Springer Science \& Business Media, 2013. 